\title{
Abstracts of the 31st Brain Impairment Conference, May 1-3, 2008, Melbourne
}

\section{Conference Theme: Brain Impairment and Ageing}

\section{PRECONFERENCE WORKSHOPS}

\section{WORKSHOP 1}

\section{Update on Neuropathology in Dementia}

Glenda Halliday

Prince of Wales Medical Research Institute, Australia

\begin{abstract}
D ecent genetic, cellular and tissue biology research has identified a relaRtively small number of protein products that form abnormal aggregates in neurodegenerative dementias. These types of research studies have also identified that different mechanisms (including genetic makeup) can trigger these aggregations. Perhaps most surprisingly, defects in gene products usually produce neurodegeneration and dementia only later in life, highlighting that longevity itself is a significant risk factor in all forms of neurodegeneration. While the delayed appearance of these dementia disorders (commonly developing over decades) is thought to be a combination of genetic and environmental susceptibilities, the reasons that different clinical disorders attack only certain sets of neurons within the brain and leave nearby neurons intact still remains largely mysterious. Despite this, by identifying the tempo of cell loss and all potential cell death mechanisms, a number of obvious therapeutic pathways become apparent. Details of the type and timing of cellular changes in the main dementia syndromes compared with normal ageing will be evaluated to highlight these concepts.
\end{abstract}

WORKSHOP 2

\section{Driving and Dementia}

\author{
Elizabeth Rand ${ }^{1}$ and Judith Charlton ${ }^{2}$ \\ ${ }^{1}$ Cognitive Dementia \& Memory Service, Caulfield General Medical Centre, Melbourne, Australia \\ ${ }^{2}$ Monash University Accident Research Unit, Australia
}

ementia will, in time, cause problems with driving. In some instances this can occur even in the very early stages of the illness. Conversely, some people are still able to drive to a safe and independent level for some time. The dilemma is defining a workable system to ensure those that can drive safely are able to continue to do so, within the law, and those that are unsafe stop. The law and current driver assessment guidelines are clear in that they state drivers have a duty of disclosure to their insurer and to the licensing authority if they have an illness that could potentially affect their driving. Older drivers self-regulate their driving; however, their ability to do so is limited when dementia is evident. Debate regarding mandatory testing of older drivers rages 
on in the media. At present the requirements for mandatory age based testing vary from state to state, and where there is a requirement for testing there is limited focus on cognitive ability. Austroad driver assessment guidelines acknowledge the need to report cognitive problems, however, the definition of which problems should be reported and when is not clearly stated. Research into the best predictors of driver safety, in particular, cognitive assessment tools, is inconclusive. At present the gold standard for assessment of driving ability (where a driver has dementia) is an occupational therapy driving assessment; however, this is costly and often stressful for the client and the availability of assessors is limited. Ideally guidelines are required to identify those that clearly should not drive (avoiding the need for an on-road assessment), those that are safe to drive and those where it is unclear (where an on-road assessment is required). In this workshop we will review the debate around older drivers with a particular focus on dementia. Dr Charlton will present evidence from her research into how age-related changes in cognition and perception influence driver behaviour and the evaluation of screening instruments for assessing functional abilities in potentially at-risk drivers. Evidence based protocols developed by the Victorian Cognitive Dementia \& Memory Services (CDAMS) to support clinicians and clients in decisions about driving will also be discussed.

\section{WORKSHOP 3}

\section{Language and Dementia}

Peter J. Nestor

Department of Clinical Neurosciences, University of Cambridge, United Kingdom

The commonest cause of dementia is Alzheimer's disease (AD) and with disease progression aphasia invariably emerges, making AD the commonest cause of language impairment in dementia. This usually takes the form of a fluent anomia; however, this deficit is only one of several cognitive problems and is characteristically less severe than impairment of memory. More important for clinical practice is aphasia as a presenting problem in a degenerative context. Progressive aphasia can be divided broadly into fluent (semantic substitutions and omissions with comprehension impairment) and nonfluent (phonological errors and hesitant speech but relatively preserved single word comprehension) forms although it is worth noting that a significant number of patients defy this strict categorisation. Fluent aphasia without prominent memory or visuospatial deficits is most classically seen in the syndrome of semantic dementia (SD) and is associated with focal degeneration of the rostral temporal lobes. SD is characteristically associated with frontotemporal dementia (FTD) pathology, most notably the variant with ubiquinated inclusions. Nonfluent progressive aphasia is also classified as a variant of FTD although this syndrome tends to be more of a mixed bag in pathological terms. Up term $40 \%$ have AD pathology, and to date, no clear linguistic or general neuropsychological factors have been reliably shown to predict this histology. In addition, a significant number evolve into a corticobasal syndrome and will have corticobasal degeneration at necropsy. Progressive supranuclear palsy can also present with prominent early loss of speech fluency usually with the hallmarks of dynamic aphasia. The workshop will focus on psycholinguistic features of progressive aphasic syndromes, neural correlates from brain imaging studies and potential clues to their pathological substrate. The presentation will include video excerpts. 


\section{WORKSHOP 4}

\section{A Case-Specific Predominantly Psychosocial Approach to BPSD}

Mike Bird

Aged Care Evaluation Unit, NSW Greater Southern Area Health Service and Australian National University, Australia

B ehavioural and psychological symptoms of dementia (BPSD) include B screaming, violence, resistance to care, repetitive questions, sexual disinhibition, and intrusive wandering. It remains a major problem in dementia care, and often leads to intolerable carer stress and the decision to institutionalise, where it causes equal distress to residential care staff. Many patients are still given antipsychotic medication, despite 15 years of meta-analyses showing modest efficacy at best and frequent side effects. Sometimes there is no alternative to psychopharmacology, but many enterprising carers or residential care staff routinely show that these behaviours can be managed without sedation, usually by providing common sense, humane care. There is also research evidence, including a controlled trial directed by the author, that careful analysis of the multiple facets of the case profile and an intervention package tailored to that profile can produce better outcomes than pharmacology. This workshop uses an interactive approach and case examples to describe how to do this. A conceptual discussion is followed by material on assessment and management of these complex problems, including alleviating the distress of those exposed to behaviour, and how to work effectively as a clinical psychologist with residential care staff. The results of several intervention studies using this approach are also presented, and recent Commonwealth and NSW initiatives discussed.

\section{INVITATION ADDRESS BY ASSBI PRESIDENT}

\section{Fantastica: The Origins of Modern Psychopharmacology}

Laurie Geffen

Professor Emeritus, University of Queensland, Australia; Psychiatrist in Private Practice

Grom the beginnings of recorded history, humans have used psychotropic substances for religious, therapeutic and recreational purposes. Lewis Lewin (1924) described 5 categories of plant-derived mind-altering drugs (excitantia, euphorica, inebriantia, hypnotica and phantastica). In this talk I will focus on phantastica and reflect on the life and scientific contributions of Lewin including his interactions with his contemporary Sigmund Freud. To the extent that all psychotropic drugs can cause brain injury, the topic is germane to the society. 
KEYNOTE ADDRESS 1

\title{
Frontotemporal Dementia: Facts, Fallacies and Fantasies
}

\author{
John R. Hodges ${ }^{1,2}$ \\ ${ }^{1}$ Prince of Wales Medical Research Institute, Australia \\ 2 University of New South Wales, Australia
}

This talk will review recent advances in frontotemporal dementia (FTD) with a brief overview of the history, epidemiology, genetics, pathology and clinical features of the three main clinical variants: behavioural variant FTD, semantic dementia (SD) and progressive nonfluent aphasia (PNFA). Of the three, SD is the most clearly defined and has informed cognitive models and the neural basis of semantic memory. The majority have ubiquitin-positive pathology. PNFA, by contrast, remains confusing: patients are heterogeneous with a range of dysarthria, speech apraxia, phonological and syntactic deficits. Those with speech apraxia are said to have insular atrophy and underlying tau-positive pathology. The application of tasks derived from developmental and social psychology has advanced the ability to diagnose and understand the clinical features of bvFTD that are associated with pathology of the ventromedial frontal lobes. In addition, with those patients who have definite structural and functional (PET) abnormalities there is also growing evidence of a nonprogressive clinical phenocopy which may represent a midlife decompensation in patients with a lifelong personality disorder or, in some cases, a neuropsychiatric syndrome.

\section{KEYNOTE ADDRESS 2}

\section{Application of a Theoretical Framework for the Delivery of Cognitive Rehabilitation for Older Healthy Adults}

\author{
Donald T. Stuss \\ Rotman Research Institute at Baycrest and Departments of Psychology and Medicine, \\ University of Toronto, Canada
}

The major objective of the presentation is to present the pros and cons of 1 the following approach to ageing: developing 'best practices' in cognitive rehabilitation requires having a sound theoretical framework that is rooted in understanding brain mechanisms. The presentation is divided into three main sections. Section 1: Focusing on memory, the inevitability of permanent deterioration with increased age is questioned. Relevant factors include the potential influence of brain plasticity, environmental contexts, and task demands. The model that appears to explain a large variance of the cognitive deterioration associated with normal ageing is one that contrasts the impact of environmental support versus self-initiated processing; that is; there is a decrease in the spontaneous use of strategic processes. This may be associated at least in part to the documented anatomical changes in the frontal lobes. Supportive evidence will be presented that highlights performance of elderly individuals on measures that have been associated with frontal lobe functioning. Section 2: Based on the hypothesis of a deficit in strategic processing in normal ageing in the context of environmental changes impacting self-attitudes, we compared two comparable normal ageing groups, one that immediately received rehabilitation and one that did not, followed by a subsequent cross-over in which the group that did not 
originally receive rehabilitation was now entered into the treatment program. The protocol consisted of three different modules, presented in the following order: memory training, practical task training, and psychosocial training. The results indicated that an emphasis on training strategic processing in the context of improved psychosocial functioning was an effective method for obtaining a sustained (tested over 6 months) increase on objective measures of memory and goal setting. Section 3: Is this good enough? Evidence will be presented that fractionates the global construct of strategic processing into distinct functions that are associated with different regions of the frontal lobes. This is consonant with developmental data indicating that different strategic processes appear at different stages in early years. We had hypothesised that there is a parallel change with ageing. That is, ageing is not a slope, but a series of slopes that may change at different ages in life. Preliminary data will be presented that support this hypothesis. What model of brain functioning - global strategic processing, or discrete and independent processing - will best serve our development of rehabilitation techniques for the normal ageing individual?

\title{
KEYNOTE ADDRESS 3
}

\section{Rapidly Progressive Dementias}

\author{
Elsdon Storey \\ Department of Neuroscience, Monash University (Alfred Hospital Campus), Australia
}

耳or the purposes of this talk, I shall arbitrarily define rapidly progressive dementias (RPDs) as those progressing to at least moderate dementia from baseline in less than 12 months. (By contrast, this typically takes several years in most degenerative dementias.) While the clinician's first thought in this scenario is usually of Creutzfeldt-Jakob disease, and this remains a very important cause of rapidly progressive dementia, there are numerous other potential causes. As a number of these are treatable, they should not be overlooked. First, the delineation between delirium and dementia begins to blur when the delirium is chronic, and it is not unreasonable to regard chronic metabolic and toxic encephalopathies as forms of dementia. Examples would include hepatic encephalopathy or lithium toxicity. Second, a number of infections may present as RPDs, including HIVADC, SSPE (in children and young adults), PML, and neurosyphilis. Third, a number of paraneoplastic and nonparaneoplastic autoimmune conditions need to be considered, including Hashimoto's encephalopathy, limbic encephalitis, and lupus cerebritis. Fourth, vascular dementias may progress rapidly: some (e.g., due to CNS vasculitis) may not do so in an obviously stepwise fashion. Fifth, it is well to remember that not all neoplasms are obvious on neuroimaging: gliomatosis cerebri and intravascular lymphoma are examples. Lastly, even typical chronic degenerative dementias such as Alzheimer's and DLB may sometimes present subacutely. A thorough diagnostic work-up is typically required, of which neuropsychological assessment forms an important part, along with clinical neurological assessment, neuroimaging (especially MRI), blood tests, and probably CSF examination and EEG. An inpatient admission is usually required to obtain the information required to diagnose a treatable cause in a timely fashion. 


\section{KEYNOTE ADDRESS 4}

\section{Issues in Mild Cognitive Impairment}

Peter J. Nestor

Department of Clinical Neurosciences, University of Cambridge, United Kingdom

6 Iild cognitive impairment' (MCI) is presently the preferred term to describe patients with cognitive problems that are too significant to be confidently attributed to normal variation, yet insufficient to fulfill diagnostic criteria for a dementia syndrome such as Alzheimer's disease (AD). The MCI criteria proposed by the Mayo clinic were expressly designed to enrich study cohorts for patients whose cognitive symptoms were likely to represent incipient dementia. The primary motive of this exercise was for clinical trials of novel therapies. Key criteria included informant corroboration of memory symptoms and objective evidence of memory impairment on formal testing. Both of these criteria reduce, but do not eliminate, the risk of including memory complainers whose symptoms do not represent the first stages of dementia. MCI is operationally separated from $\mathrm{AD}$ by stipulations that patients have preserved living activities, are not 'demented', and do not fall into a predetermined dementia range on cognitive test batteries. Combining these elements it is straightforward to set out very precise 'MCI' inclusion criteria for a research trial. For instance, hypothetically, one could proscribe (a) patient and spouse confirm progressive memory symptoms (b) scores worse than 2 standard deviations below matched-control mean on delayed recall of the Jumbo-Memory Words and Designs Test (c) can dress, wash, use a toilet and prepare a cup of coffee independently and (d) scores $\geq 26 / 30$ on the Universal Global Cognition Examination. Problems start to emerge with the MCI concept; however, when one does not stipulate precise criteria this immediately leads to problems with definition of each criterion. For example, Which memory test defines impairment? Which global measure and cut-off score defines absence of dementia? Are daily living activities preserved if the patient can dress but never remembers to change their clothes? Introduction of the MCI concept into clinical practice can be even more problematic. The transition from MCI to 'probable AD' is insidious and difficult to define with precision in individuals. It is also important to remember that this transition is meaningless in pathological terms. When patients are said to 'convert' from MCI to probable AD, the 'conversion' is only from one arbitrary set of clinical criteria to another - the individual always had AD pathology. Although this statement should be obvious, it is easy to overlook in clinical practice. Rigid adherence to a predefined set of MCI criteria can run the risk of both increasing anxiety in the worried-well (applying the MCI label to patients whose symptoms are almost certainly benign) and of artificially delaying the diagnosis of AD (holding patients in an 'MCI limbo' when clinical information is showing a progressive syndrome qualitatively typical of that seen in AD). 


\section{KEYNOTE ADDRESS 5}

The Australian Imaging Biomarkers and Lifestyle Flagship Study of Ageing

David Ames

University of Melbourne, National Ageing Research Institute, Australia

This CSIRO funded study involves the collaboration of numerous Pelbourne and Perth. It aims to recruit 200 individuals with mild Alzheimer's disease (AD), 200 with mild cognitive impairment (MCI) and 600 healthy aged controls of whom at least 200 will express concern about their memory performance. Baseline assessments consist of neuropsychological testing (see Greg Savage's presentation at this meeting for more detail), drawing of $80 \mathrm{ml}$ blood for biomarker analysis and completion of a health and lifestyle questionnaire. 250 members of the cohort will undergo PIB PET brain scans to assess brain amyloid burden. All participants will be seen again and reexamined as per baseline protocol (with the exception of the health and lifestyle questionnaire) after 18 months. As of January 11, 2007, 759 subjects had been recruited and over 116 had undergone PIB scans. It is hoped that viable markers of presymptomatic AD will be discovered. Initial PIB results indicate a correlation of amyloid load with cognitive impairment in MCI and normal subjects and very high levels of amyloid burden in AD patients.

\section{INVITED SPEAKER 1}

\section{Traumatic Brain Injury and Older Adults}

Glynda Kinsella

Psychological Science, La Trobe University and Psychology, Caulfield General Medical Centre, Melbourne, Australia

\footnotetext{
s positive health trends have led to a healthier and more mobile older A population, older adults are now at greater risk of sustaining trauma through road traffic accidents or falls. Traumatic brain injury (TBI), a frequent consequence of traumatic injury, is commonly associated with young males, however, older adults (65 years+) form the second most frequently injured demographic, and yet very few studies have focused on older adults. Instead of assuming that outcome posttrauma will necessarily follow similar patterns as in a younger sample, there are several reasons to investigate recovery separately for an older population: (a) mechanism of the injury, (b) effects of cognitive ageing, (c) comorbid health conditions, and (d) emotional response to trauma. Taken together, there is a strong argument for investigating the differential response and recovery patterns of older adults to TBI and, within the context of a critical review of the existing research, some preliminary findings from a Victorian study will be presented.
} 


\title{
INVITED SPEAKER 2
}

\section{The Kimberley Indigenous Cognitive Assessment (KICA): Development of a Cognitive Tool for Elderly Indigenous in Remote and Rural Areas, and Determination of Prevalence of Dementia in This Region}

\author{
D. LoGiudice, ${ }^{1}$ K. Smith, ${ }^{2}$ L. Flicker, ${ }^{2}$ O. Almeida, ${ }^{2}$ N. Lautenschlager, ${ }^{2}$ A. Dwyer ${ }^{2}$ \\ and D. Atkinson ${ }^{3}$ \\ ${ }^{1}$ Royal Park Campus, Royal Melbourne Hospital, Parkville and National Ageing Research Institute, \\ Parkville, Australia \\ 2 University of Western Australia, Australia \\ ${ }^{3}$ Kimberley Aboriginal Medical Services Council, Broome, Australia
}

This presentation will describe the planning, development and validation of a culturally appropriate tool for older Indigenous people in the Kimberley. The KICA was developed with Indigenous health and aged care organisations. It comprises of medical history, cognition, depression, and carer report of daily function. Validity was tested in 70 Indigenous subjects over 45 years of age. Clients and carers were assessed by the KICA and then independently assessed using DSM-IV and ICD-10 criteria. Interrater reliability was assessed in 14 subjects rated by two independent observers. Five items on the cognitive score (orientation, naming, registration, recall and free recall) effectively discriminated $91.4 \%$ of cases. Sensitivity and specificity was $91 \%$ and $93 \%$, respectively, using a cut off score of 31-32/39. The KICA is now widely used in remote and rural areas of Australia. Following the development of the KICA, 363 participants (aged over 45 years) selected from 6 Aboriginal communities and 1 town were assessed with this tool to determine the prevalence of dementia and associated risk factors amongst older Indigenous Australians living in the Kimberley. All participants with low cognitive scores, as well as a random sample of those with normal results in the KICA, were reviewed by a geriatrician or old age psychiatrist who was blind to the results derived from the KICA. Consensus diagnoses were made by two specialists in geriatric medicine or old age psychiatry using ICD-10 criteria. The mean age of participants was $60.7 \pm 11.9$ years and $55 \%$ were women. The prevalence of dementia was $12.4 \%, 4.8$-fold greater than the overall Australian prevalence. Males were more likely to have dementia than females ( $17 \%$ vs. $9 \%$; OR $3.2,95 \%$ CI $1.4,6.9)$. Other significant risk factors for dementia in this sample included older age, no formal education (OR 2.7, 95\% CI 1.1, 6.8), current smoking (OR 3.1 95\% CI 1.0, 9.5), previous stroke (OR17.1, 95\%CI 5.9, 49.7), head injury (OR $4.1,95 \%$ CI 1.6, 10.6) and epilepsy (OR 47.4, 95\% CI 6.0, 369.8). Other measured factors associated with dementia included falls, incontinence and poor mobility. The significant risk factors for cognitive impairment were age, male gender, previous stroke, epilepsy and head injury. The KICA appears to be a valid and reliable assessment tool for cognitive impairment in an older Indigenous population. Indigenous Australians living in the Kimberley have a much higher prevalence of dementia than previously reported for nonIndigenous Australians, particularly in the younger age groups. Risk factors associated with dementia in this sample are likely to reflect the underlying pathophysiological mechanisms that contributed to the development of dementia in this remote Indigenous community. 


\title{
INVITED SPEAKER 3
}

\section{The Basal Ganglia Circuits, Dopamine and Language: Insights from Studies in Parkinson's Disease}

Helen Chenery

Faculty of Health Sciences, University of Queensland, Australia

\begin{abstract}
Darkinson's disease, while traditionally thought of as primarily a movePent disorder, also encompasses changes to both cognitive (including linguistic) processes. Of interest is the role of the various subcortical circuits in these non-motor processes and how Parkinson's disease (PD) leads to changes in some but not all of the basal ganglia thalamocortical circuits. In this paper, the results of various language experiments are presented from people who have undergone deep brain stimulation (DBS) of the subthalamic nucleus (STN) for treatment of their medically intractable PD. Participants were tested when their stimulators were turned on and when they were turned off. The results suggest that STN stimulation improves cognitive-linguistic processes that are more dependent upon anterior cingulate circuits, yet results in a deterioration of performance on tasks thought to rely upon the dorsolateral prefrontal circuit. The implications of these findings for the management of people with PD post-DBS will be discussed.
\end{abstract}

\section{INVITED SPEAKER 4}

\section{Anxiety in the Elderly - The Geriatric inventory (GAI): Development Characteristics and Use in Neuropsychological Populations}

Nancy Pachana

School of Psychology, University of Queensland, Australia

\begin{abstract}
nxiety disorders are more common in later life than depressive disorders, and are often difficult to distinguish from a variety of medical and neurological disorders, yet clinical inventories measuring anxiety symptoms in this population have been lacking. The Geriatric Anxiety Inventory (GAI), a brief measure of dimensional anxiety specifically designed for use in older adults, was developed and validated in a variety of patient populations. This 20 -item scale has shown good reliability in both normal community and psychiatric samples (Cronbach's alpha 0.91 and 0.93, respectively). Concurrent validity with other measures was high, while interrater and test-retest reliability were excellent. The ability of the GAI to discriminate between patients with and without any anxiety disorder, $F(1,44)=16.87, p=.0002$, was also excellent. Receiver operating characteristic (ROC) analysis indicated an optimum cut point of $8 / 9$ to identify older patients with any anxiety disorder, correctly classifying $78 \%$ of patients (sensitivity $73 \%$, specificity $80 \%$ ). The GAI is in use in five countries, with evidence of good pre-post utility in treatment studies on clinical populations. Examples of the use of the GAI in neurological populations of interest, including persons with mild cognitive impairment and Parkinson's disease, will be given.
\end{abstract}




\section{INVITED SPEAKER 5}

\section{Science, Law and the Assessment of Decision-Making Capacity}

Elizabeth Mullaly

Caulfield General Medical Centre, Australia

6 ou will realise by now that value judgments come into medical decision making. You will face situations where the right decision is unclear, you have to use your judgement and evidence-based medicine won't help you' (The Ethox Centre, Department of Public Health and Primary Health Care, University of Oxford, Ethics and Law teaching materials, 2005). One potential consequence of dementia and other forms of brain impairment is the loss of capacity to make decisions about finances, healthcare and accommodation. However, determining the threshold for such incapacity is complex and challenging, in part due to the lack of an evidence-based approach that provides a scientific basis for practice. The issue of validity in relation to capacity assessment methods will be discussed, as well as some recent court decisions that shed light on legal approaches when clinician opinion varies.

\section{INVITED SPEAKER 6}

\section{The Natural History of Akinetic Rigid Syndromes}

David R. Williams

Alfred Hospital, Cabrini Hospital, Faculty of Medicine (Neurosciences), Monash University (Alfred Hospital Campus), Australia

Veurodegenerative Parkinsonism, characterised by tremor, rigidity, 1 bradykinesia and postural instability, is frequently accompanied by other clinical features, indicative of widespread neuronal dysfunction. In the classic descriptions of Parkinson's disease, progressive supranuclear palsy, multiple systems atrophy and corticobasal degeneration the movement abnormalities have been emphasised and the cognitive features have been overlooked. Since these early reports a clearer understanding of the progressive cognitive decline that occurs in these patients has developed. More recently, studies of the natural history of these conditions suggest that clinical and cognitive features can predict disease course, prognosis and the topographical distribution of pathology.

\section{INVITED SPEAKER 7}

\section{Prevalence, Incidence and Issues for Diagnosis of Mild Cognitive Disorders in Young-Old Adults}

Kaarin J. Anstey

NHMRC and Ageing Research Unit, Centre for Mental Health Research, Australian National University, Australia Prevalence and incidence rates of mild cognitive impairment and related
disorders, were examined in The PATH Through Life Study. The baseline assessment in 2001-2002 included 2551 60- to 64-year-olds with 2222 participating in a 4-year follow-up assessment. Participants were screened 
with the MMSE and a short cognitive battery. Those screened positive received clinical assessment for diagnoses of mild cognitive disorders or dementia using established clinical criteria. Prevalence and incidence rates for the cohort were estimated with predictive regression models. The annual incidence of dementia was $0.25 \%$. The prevalence of mild cognitive impairment was $4.2 \%$, age associated memory impairment was $2.4 \%$, age-associated cognitive decline was $7.6 \%$, mild neurocognitive disorders was $12.87 \%$ and other cognitive disorder was $7.3 \%$. The prevalence of any diagnosis of any mild cognitive disorder (Any-MCD) was $29.5 \%$ and the annual incidence rate for AnyMCD was $5.7 \%$. Agreement for specific diagnoses between Waves 1 and 2 was fair to poor (0 to $47.0 \%$ ) but agreement for Any-MCD over 4 years was $89.0 \%$. The MMSE and a word recall test reliably detected the presence of any mild cognitive disorder in young-old adults. A range of health and lifestyle factors was examined as predictors of conversion to MCI. We conclude that diagnoses of mild cognitive disorders do not predict dementia at 4-year followup in young-old adults. Prevalence rates for mild cognitive disorders vary greatly depending on criteria and time of assessment. However, diagnosis of Any-MCD is relatively stable in young-old adults.

\section{INVITED SPEAKER 8}

\section{Beta-Amyloid Imaging in Dementia}

Christopher Rowe ${ }^{1,2}$

${ }^{1}$ Department of Nuclear Medicine and Centre for PET, Austin Health, Australia

${ }^{2}$ Department of Medicine, University of Melbourne, Australia

B eta-amyloid imaging with positron emission tomography (PET) proB vides an important new tool for the evaluation of the causes, diagnosis and future treatment of dementias. Beta-amyloid imaging should allow earlier diagnosis of Alzheimer's disease (AD) and accurate differential diagnosis of the dementias. Studies with $11 \mathrm{C}-\mathrm{PiB}$ and other beta-amyloid tracers show robust cortical binding in almost all AD subjects, and correlate well with reduction in CSF A-beta-42. Binding also correlates with the rate of cerebral atrophy as measured by magnetic resonance imaging (MRI) and with episodic memory impairment in apparently normal elderly individuals and in subjects with mild cognitive impairment. Increased binding is predictive of conversion of mild cognitive impairment to AD. Comparison of betaamyloid imaging versus 18F-fluorodeoxyglucose (FDG) PET demonstrates greater accuracy for distinguishing subjects with mild AD from elderly controls. Subsequent to recent advances in imaging and CSF analysis, it has been proposed that the research criteria for the diagnosis of probable AD should be revised to allow earlier diagnosis and therapeutic intervention. It is argued that dementia is not required for the diagnosis of $\mathrm{AD}$ when there is a clear history of progressive cognitive decline, objective evidence from psychometric tests of episodic memory impairment and characteristic abnormalities in the CSF or in neuroimaging studies. The latter includes the demonstration of beta-amyloid with appropriate PET tracers. Thus, as the criteria for the diagnosis of $\mathrm{AD}$ evolve, and new therapies for $\mathrm{AD}$ emerge, beta-amyloid imaging is likely to have an increasingly important role in clinical practice provided it is accessible and affordable. 


\title{
The Relationship Between Age and MRI Findings Following Traumatic Brain Injury
}

\author{
Michael Schönberger ${ }^{1,2}$ and Jennie Ponsford ${ }^{1,2,3}$ \\ ${ }^{\prime}$ School of Psychology, Psychiatry and Psychological Medicine, Monash University, Australia \\ ${ }_{2}^{2}$ Monash-Epworth Rehabilitation Research Centre, Epworth Hospital, Melbourne, Australia \\ ${ }^{3}$ National Trauma Research Institute, Melbourne, Australia
}

\begin{abstract}
number of studies have shown a relationship between age and cognitive A and psychosocial outcome. The aim of the current study is to investigate whether this relationship is reflected in the relationships between TBI individuals' age, injury severity, time since injury and viable brain volume as well as lesion size on MRI. The study is ongoing. Design: In a cross-sectional design, MRI scans of to date 73 TBI survivors (56 males, 17 females, mean age at MRI scan 37.5 years, $S D=13.1$, range 19-77) have been examined in relation to their age, gender, PTA duration and, time from injury to MRI scan. Logistic regression analysis shows that presence of larger brain lesions is associated with older age, longer PTA and longer time since injury. Age and PTA interact in the prediction of large lesions. Findings confirm that PTA is a valid measure of injury severity, given its significant association with lesion size. They also suggest that those who are older are more susceptible to injury, having larger lesions on MRI. This could explain the poorer outcomes frequently observed in older age groups following TBI.
\end{abstract}

\section{SYMPOSIUM PAPER 2}

\section{The Effect of Age on Long-Term Cognitive and Emotional Outcome Following Traumatic Brain Injury}

\author{
Dawn Senathi-Raja, ${ }^{1.2}$ Jennie Ponsford ${ }^{1.2 .3}$ and Michael Schönberger ${ }^{1.2}$ \\ ${ }^{1}$ School of Psychology, Psychiatry and Psychological Medicine, Monash University, Australia \\ ${ }^{2}$ Monash-Epworth Rehabilitation Research Centre, Epworth Hospital, Melbourne, Australia \\ ${ }^{3}$ National Trauma Research Institute, Melbourne, Australia
}

t has been suggested that as the brain ages following injury, cognitive
recovery may occur to a lesser extent due to a finite capacity of the brain
for plasticity. On the other hand, it is arguable that older age at the time of
traumatic brain injury (TBI) may militate against emotional problems due to
preinjury attainment of life goals. Previous studies examining effects of age
on long-term outcome have been limited by poor differentiation between the
normal ageing process and injury-related decline. The objective of this study
is to investigate whether, in the context of normal aging, individuals sustain-
ing TBI at an older age show poorer cognitive recovery but better emotional
outcome than patients who were younger at the time of injury. Using a
prospective study design, 112 head injured patients ( $59 \%$ males) aged
between 26 and 89 years $(M=54.88, S D=17.54)$ who had sustained mild 
to severe TBI were followed up a mean of 11 years post-injury. The control group comprised 112 healthy individuals each matched to a TBI participant in terms of gender, age, and estimated IQ. A range of cognitive measures was used to assess processing speed, attention, memory and executive function. Emotional adjustment was measured using the Hospital Anxiety and Depression Scale. Detailed study findings will be presented. Preliminary results suggest a trend for older TBI patients to be disproportionately impaired on certain speed and memory measures. On the other hand, anxiety and depression levels appear to be relatively lower in the oldest patients relative to younger and middle aged patients. The findings of this study have implications for the planning and provision of appropriate rehabilitation and long-term supports for patients at different life stages.

\title{
SYMPOSIUM PAPER 3
}

\section{Effects of Age on Long-Term Outcome Following TBI}

\author{
Jennie Ponsford ${ }^{1,2}$ \\ ${ }^{1}$ School of Psychology, Psychiatry and Psychological Medicine, Monash University, Australia \\ ${ }^{2}$ Monash-Epworth Rehabilitation Research Centre, Epworth Hospital and National Trauma Research \\ Institute, Melbourne, Australia
}

\begin{abstract}
ge has been identified as a factor which significantly influences outAcome following traumatic brain injury (TBI). However, this operates in a complex fashion. The influence of age is potentially modified by environmental and developmental factors. This paper will examine the impact of age on functional and psychosocial outcome two years following TBI. Participants include a cohort of 808 patients with moderate to severe TBI, aged 15 to 80, treated at Epworth Rehabilitation Centre and followed up 2 years postinjury. They completed measures including a Structured Outcome Questionnaire covering independence in ADL, vocational and relationship status and changes in cognitive, behavioral and emotional function, the Craig Handicap Assessment and Reporting Technique (CHART), Glasgow Outcome Scale-Extended (GOS-E) and Hospital Anxiety and Depression Scale. Univariate comparisons indicated that those who were older (age > 45) showed significantly worse outcomes in the domains of mobility, independence in ADL and employment, and lower scores on the GOS-E and all CHART subscales than those aged $<45$ years. These differences were also evident in another cohort of 170 moderate-severe TBI patients followed up on the same measures at 10 years postinjury, but those who were younger were more likely to report being affected from a cognitive, psychosocial and emotional point of view. These findings demonstrate poorer functional outcomes in those who are older at injury. However, the association between age and psychosocial outcome is far more complex with older persons less disadvantaged. The impact of the ageing process on TBI individuals has yet to be documented.
\end{abstract}




\title{
SYMPOSIUM PAPER 4
}

\section{The Relationship Between Age and Employment Outcome After Traumatic Brain Injury: What Are the Mediating Variables?}

\author{
Michael Schönberger ${ }^{1,2}$ and Jennie Ponsford 1,2,3 \\ ${ }^{1}$ School of Psychology, Psychiatry and Psychological Medicine, Monash University, Melbourne, Australia \\ ${ }^{2}$ Monash-Epworth Rehabilitation Research Centre, Epworth Hospital, Melbourne \\ ${ }^{3}$ National Trauma Research Institute, Melbourne, Australia
}

$\mathrm{T}$

The frequently established relationship between age and work integration after traumatic brain injury (TBI) may be caused by complex interactions between a range of biological and psychosocial factors. The aim of the current study was to investigate how the process of work integration after TBI is related to age at injury, and whether this relationship can be explained by patients' demographic and injury characteristics. The study is ongoing. Length of PTA, type of accident, premorbid employment status and education of to date 328 individuals with moderate to severe TBI (69\% males, $31 \%$ females, mean age at injury 34 years, $S D=17$, range 14-79), was registered at the time of injury. Work integration was measured one and five years postinjury. The pathway of work integration depended on patients' age, with patients aged 20 to 34 showing the best work integration. Both younger and older patients had poorer outcomes. The age group 20-34 had the second-shortest PTA, the lowest percentage of pedestrians hit by vehicles, the highest level of education, the highest percentage of preinjury employment and the highest percentage of males, all of which were related to good work integration. Demographic and injury-related variables mediate the effect of age on employment outcome. The mediating variables might be related to age for several reasons; for example, many teenagers were still in education and might have been involved in more severe car accidents; for elderly persons, the lower education might be a cohort effect, while severity of injury might be related to reduced recovery potential.

\section{CONCURRENT SESSION 2: UNUSUAL DEMENTIAS}

INVITED SPEAKER 2 (DINA LOGUIDICE)

\section{PAPER 1}

\section{Rapidly Progressive Dementia: A Case Report and Literature Review}

Ramy Ghaly, ${ }^{1}$ Ronald Leong ${ }^{2}$ and Simon Scharf ${ }^{2}$

${ }^{1}$ Eastern Health Service, Box Hill Hospital \& Peter James Centre, Australia

2 Caulfield General Medical Centre, Australia

$\boldsymbol{B}_{\text {ackground: We report a case of a } 70 \text {-year-old woman who presented }}$ $\boldsymbol{B}_{\text {with a subacute progressive dementia associated with visual hallucina- }}$ tions, pyramidal and extrapyramidal features, insomnia and weight loss. Brain SPECT showed reduced bi-parietal perfusion. CSF analysis revealed atypical protein 14-3-3; a pattern which neither excluded nor confirmed a diagnosis of Creutzfeldt-Jakob disease (CJD). EEG was negative for periodic synchronous sharp wave complexes. Antemortem diagnosis was a mixed dementia; probable Alzheimer's disease (AD) and dementia with Lewy Bodies (DLB). Rapidly progressive dementia (RPD) is an unusual 
form of dementia, often with a sub-acute course and atypical clinical features. Diagnostic algorithms for RPD are not well established and this syndrome is challenging to clinicians. Objective: A literature review to formulate a diagnostic approach to RPD with a focus on neurodegenerative, autoimmune and neoplastic aetiologies. Methods: Search via Medline database. Keywords: 'rapidly progressive dementia'. Results: CJD is one of the prototypical causes of RPD. Sporadic CJD (sCJD) accounts for $85 \%$ of cases of CJD. Detection of protein 14-3-3 in CSF has an average sensitivity and specificity of $92 \%$ as a diagnostic biomarker for sCJD. EEG criteria have a positive and negative predictive value of $95 \%$ and $49 \%$ respectively. Rapidly progressive $\mathrm{AD}$ is the most frequent alternative diagnosis followed by DLB. Autoimmune disease and neoplasms are uncommon causes for RPD, however, should be considered as they may be partially reversible. Conclusion: While CJD remains the primary diagnosis for exclusion in RPD, diagnostic algorithms need to be broad enough to include alternative, potentially treatable causes.

\title{
PAPER 2
}

\section{Multiple Sclerosis: Related Dementia as an Unusual Presenting Feature}

\author{
Wendy A. Longley and Gary Fulcher \\ Multiple Sclerosis, NSW/VIC, Australia
}

\begin{abstract}
Tn January 2002, 36-year-old Doug was accidentally hit on the head by a 1 metal bar while working as a fitter and turner. He suffered no loss of consciousness and continued working. Two days later he began to lose vision in his left eye. Subsequent investigations, including MRI, lead to a diagnosis of multiple sclerosis (MS). Although his vision impairment improved with treatment, within a few weeks he started developing marked memory and personality changes. He was hospitalised, and was seen by a psychiatrist who described him as "slightly fatuous, dependent, impulsive, with poor attention, self-monitoring and judgment as well as difficulties with memory, verbal fluency, perseveration, emotional blunting and cognitive rigidity'. A repeat MRI in April showed a marked increase in white matter signal intensity throughout many regions of the brain, but particularly in both frontal lobes. Although it was hoped that this was a temporary 'exacerbation', these cognitive and behavioural features only abated slightly, and a neuropsychological assessment a year later confirmed persistent widespread cognitive impairments consistent with MS-related dementia (Longley, 2007). Laurie's wife, Carla, enquired whether they could claim workers compensation for the workplace accident that had 'caused' Doug's MS. This presentation includes the rest of Doug and Carla's story, a short review of MS-related dementia, including dementia as an unusual presenting feature, a brief review of the current theories regarding the aetiology of MS, and an answer to the question about whether physical trauma can cause MS.
\end{abstract}




\title{
CONCURRENT SESSION 3: WHAT'S NORMAL IN AGEING?
}

\section{PAPER 1}

\section{Do Older People Forget? Memory Performances in the Normal Aged}

T. Wardill ${ }^{1}$ and V. Anderson ${ }^{2,3}$

${ }^{1}$ Melbourne Neuropsychology Service, Australia

2 University of Melbourne, Australia

${ }^{3}$ Murdoch Children's Research Institute, Australia

\begin{abstract}
$\mathrm{O}$ ne impact of our ageing population is an increased demand for clinicians to accurately assess and diagnose cognitive disorders in older people. The assessment of memory plays a crucial role in any cognitive assessment. Memory disturbance can accompany many of the common diseases seen in the aged and may be the first indication of pathology. If clinicians are to accurately detect memory dysfunction in older adults, an understanding of normal memory performance in the aged is required. This paper will present data from a study on the cognitive performance of older adults. Data were obtained from a large sample of carefully screened Australians in the age range 65 to 94 years. The performances of the normal aged sample on a range of commonly used clinical measures of memory will be presented, and compared to those seen in one of the common disorders of ageing, dementia of the Alzheimer's type. These data reveal valuable information about the impact of the ageing process on memory, and provide clinicians with useful tools for distinguishing between normal and pathological ageing.
\end{abstract}

\section{PAPER 2}

\section{Use of the Arizona Battery for Communication Disorders of Dementia (ABCD) to Differentiate Profiles Associated With Mild Alzheimer's Disease, Normal Ageing and Major Depression}

Jacinta Douglas ${ }^{1}$ and Bronwyn Moorhouse ${ }^{2}$

1 School of Communication Sciences, La Trobe University, Australia

2 Royal Talbot Rehabilitation Centre, Austin Health, Victoria, Australia

\begin{abstract}
A ccurate and early assessment of dementia is important not only in terms of meeting the needs of those with dementia, but also in identifying those who present with disorders like depression, that share similar symptoms. Language assessment plays a valuable role in assisting the differential diagnosis process. This study was designed to compare communication profiles associated with normal ageing, mild Alzheimer's disease (AD) and major depression. A total of 33 individuals participated in the study: 11 normal elderly (mean age $78.6 \pm 10.6), 11$ with mild $\mathrm{AD}(74.5 \pm 7.6)$ and 11 with major depression (70.8 \pm 9.5 ). The assessment protocol included Mini Mental Status Examination (MMSE), Geriatric Depression Scale, National Adult Reading Test (NART) and the ABCD. Group differences were evaluated using ANOVA with multiple comparisons and an adjusted alpha level of .01. Scores of both the normal elderly and the depression groups were significantly higher than those of the mild AD group on 9/17 ABCD subtests. The only subtest to yield a significant difference between the normal elderly and the depressed groups was the generative naming (semantic verbal fluency) subtest. The performance of the normal elderly on this subtest was significantly superior to
\end{abstract}


that of the depressed group, whose performance did not differ significantly from that of the mild AD group. Previous findings have supported the use of the $\mathrm{ABCD}$ in differentiating normal and dementia-related communication and linguistic function. The results of this study further support the sensitivity of the measure, in this case with respect to differentiating depression-related versus dementia-related performance.

\title{
PAPER 3
}

\section{Postmortem Findings of White, Rather Than Grey, Matter Loss in Healthy Ageing and Relation to Cognition}

Olivier Piguet, ${ }^{1}$ Jillian J Kril² and Glenda M Halliday ${ }^{1}$

${ }^{1}$ Prince of Wales Medical Research Institute, Australia

2 Disciplines of Medicine and Pathology, The University of Sydney, Australia

\begin{abstract}
A ge-related changes in total and regional grey and white matter brain volumes are widely documented. The reported effects of age, however, are much more pronounced and widespread in neuroimaging than in postmortem studies, with the largest changes described in the prefrontal cortex and the middle temporal lobe region. Because of differences in measurement methods and case selection between neuroimaging and postmortem studies, the magnitude of the effect that is specific to chronological age remains unresolved. We carried out postmortem volume measurements for 26 cortical, subcortical and white matter regions, in 24 human brains (13 men) aged 46 to 92 years, free of neuropathological abnormalities. Significant agerelated loss was observed in anterior and posterior white matter but not in total grey matter volumes. Further analyses on 5 cortical subregions previously reported to exhibit large age-related loss on MRI yielded negative results. These analyses demonstrate smaller changes with age than those reported in imaging studies. This discrepancy between postmortem and imaging studies may partly be explained by the increase in noise of the neuroimaging data with age. We discuss our findings in relation to the age-associated cognitive changes reported in the literature, and in the view of recent neuroimaging (diffusion tensor imaging) data. Conclusion: Our results suggest that healthy brain ageing is a process affecting predominantly white matter not grey matter.
\end{abstract}

\section{PAPER 4}

\section{Discovering the Early Biomarkers of Alzheimer's Disease: Neuropsychological Findings from the Longitudinal AIBL Flagship Study of Ageing}

\author{
G. Savage, ${ }^{1}$ D. Ames, ${ }^{2}$ C. Masters, ${ }^{3,4}$ R. Martins, ${ }^{5}$ T. Lockett ${ }^{6}$ and K. Ellis ${ }^{2}$ \\ ${ }^{1}$ Macquarie Centre for Cognitive Science (MACCS), Macquarie University, Australia \\ ${ }^{2}$ Department of Psychiatry, University of Melbourne, Australia \\ ${ }^{3}$ Centre for Neurosciences, University of Melbourne, Australia \\ ${ }^{4}$ The Mental Health Research Institute of Victoria, Australia \\ ${ }^{5}$ SEBHS, Edith Cowan University, Australia \\ ${ }^{6}$ CSIRO, Australia
}

\footnotetext{
The Australian Imaging Biomarkers and Lifestyle (AIBL) Study of Ageing is a 3-year longitudinal cohort project which aims to improve understanding of the pathogenesis and diagnosis of Alzheimer's disease
} 
(AD). Neuropsychological, neuroimaging and biomarker techniques are employed, and the study includes an examination of lifestyle and dietary factors associated with $\mathrm{AD}$ and healthy ageing. The cohort will comprise 1000 volunteers (minimum age 60 years) with 200 participants from each of the following 5 groups: mild $\mathrm{AD}$, mild cognitive impairment, healthy agedmatched controls (ApoE4+), healthy aged-matched controls (ApoE4-), and 'memory complainers' (healthy volunteers reporting subjective memory complaints). At baseline and 18-month timepoints all participants undergo clinical/neuropsychological assessment and blood biomarker analysis. A subgroup (25\% of the cohort) also undergo imaging ([C-11]PIB-PET and structural MRI). All participants complete questionnaires assessing diet and exercise patterns, and a subgroup receive actigraph accelerometer measurement of activity levels and dual energy X-Ray absorptiometry measures of body composition. This is the largest study of its kind ever undertaken in Australia. Since recruitment began in October 2006 we have telephonescreened 2000 volunteers for eligibility and recruited 1600 who passed the inclusion/exclusion criteria and are willing to participate at our Melbourne and Perth testing sites. One year later we have baseline-tested 482 participants (mean age 72 years, range 60-95) and undertaken 81 imaging studies. The AIBL study is on track to complete baseline testing by early to mid2008 , and the presentation will outline the principal findings of the available data set.

\title{
CONCURRENT SESSION 4: MOVEMENT DISORDER DEMENTIAS
}

\section{INVITED SPEAKER 3 (HELEN CHENERY)}

\section{PAPER 1}

\section{Cortical and Cognitive Changes in Presymptomatic Huntington's Disease}

\author{
J.W. Davison, ${ }^{1}$ L.J. Tippett, ${ }^{1}$ V. Hogg, ${ }^{1}$ R. Roxburgh ${ }^{2}$ and H.D. Rosas ${ }^{3}$ \\ ${ }^{1}$ University of Auckland, New Zealand \\ ${ }^{2}$ Auckland City Hospital, New Zealand \\ ${ }^{3}$ Massachusetts General Hospital/Harvard University, United States of America
}

Uuntington's disease (HD) is a hereditary neurodegenerative disease leading to profound neurological deficit characterised clinically by a triad of motor, cognitive and affective impairment. Although HD is a relatively low frequency disease, it is an excellent 'model' of neurodegenerative disease, because its mechanisms are relatively well understood. It has been generally accepted that most of the clinical and neuropsychological difficulties experienced in HD are related to degeneration of the striatum, both directly, and as a result of disrupted functioning of frontal-striatal anatomical loops. However, in a recent imaging study Rosas et al. (2002) found that cortical thinning occurred early in the disease, and surprisingly, was most prominent over posterior cortical regions in the early stages. With increased disease severity cortical thinning progressed to include anterior cortical regions. Few studies have investigated functions associated with posterior cortical regions in individuals with early HD. The aims of this study were first, to replicate Rosas et al.'s findings of posterior cortical thinning in people with presymptomatic HD employing identical MRI-based methods; and second, to investigate whether posterior cortical thinning is correlated with 'posterior' cognitive impairments. To achieve the second aim a number of neuropsychological tasks were used that 
were sensitive to specific cognitive abilities known to be subserved predominantly by the posterior regions of the brain. The MRI images and neuropsychological test scores of 20 participants with presymptomatic HD was compared with 20 age, gender, and education-matched controls with no risk of developing HD. Results will be presented.

\title{
PAPER 2
}

\section{The Identification of Mild Cognitive Impairment in Parkinson's Disease}

\author{
A. McKinlay, ${ }^{1}$ R.C. Grace, ${ }^{1}$ J.C. Dalrymple-Alford ${ }^{1,2}$ and D. Roger $^{1}$ \\ ${ }^{1}$ Psychology Department, University of Canterbury, New Zealand \\ ${ }^{2}$ Van Der Veer Institute for Parkinson's and Brain Research, Christchurch, New Zealand
}

\begin{abstract}
Cognitive deficits are common for Parkinson's disease patients (PD), but only a subgroup will progress to dementia (PDD). Unfortunately, specific cognitive deficits that signal preclinical dementia in PD remain unknown, making intervention difficult. Forty PD patients without signs of dementia were assessed on a wide range of neuropsychological tests and cognitive domains. Cluster analysis was then used to identify different subgroups of patients. These subgroups were then assessed in terms of betweengroup differences and also compared to individually matched healthy controls in cognitive functioning and ability to conduct activities of daily living. Three subgroups of patients were identified that formed a continuum of cognitive impairment from none/mild to severe. Compared to controls, 1 subgroup showed no or minimal cognitive impairment (PD-NCI), a second group showed an uncertain pattern of mild to severe impairments (PD-UCI), and a third group had evidence of severe impairment across most of the cognitive domains tested. This latter group was labeled PD-mild cognitive impairment (PD-MCI). The PD-UCI and PD-MCI groups were also significantly different from their controls with respect to their ability to carry out functional activities of everyday living. PD patients are heterogeneous with regard to their cognitive presentation and ability to carry out daily activities. Moreover, deficits were unrelated to other clinical and demographic characteristics. This line of research has considerable clinical utility as it may enable the development of diagnostic criteria for preclinical dementia specific to PD patients (PD-MCI), providing a basis for early intervention that could slow the development of dementia.
\end{abstract}

\section{CONCURRENT SESSION 5: TRAUMATIC BRAIN INJURY}

\section{INVITED SPEAKER 4 (NANCY PACHANA)}

\section{PAPER 1}

\section{The Role of Cognitive and Affective Factors in the Development of Subjective Cognitive Impairment after a Mild Traumatic Brain Injury}

L.A. Clarke and J.F.I. Anderson

University of Melbourne, Australia

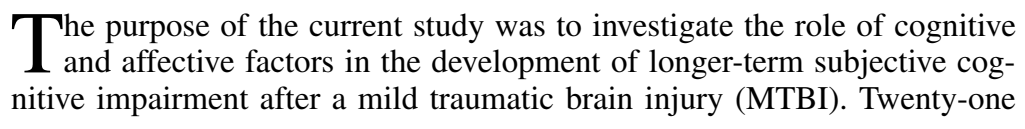


people who had sustained a MTBI in the preceding 3 to 12 months and 19 who had sustained a traumatic spinal injury (Spinal) in the same time period each completed a subjective assessment of their postinjury cognitive impairment and postconcussion symptomatology. Objective assessment of cognitive functioning, anxiety, depression, neuroticism and conscientiousness was then undertaken. The MTBI group had a significantly greater level of subjective cognitive impairment than the Spinal group. The groups did not differ significantly in their performance on any of the cognitive tests, nor in their levels of anxiety, depression, neuroticism or conscientiousness. Group means for all measures were within the 'normal' range. Anxiety and performance on the Symbol Digit Modalities Test predicted subjective cognitive impairment. The findings suggest that both anxiety and processing speed impairments may play a role in the development of subjective cognitive impairment after a mild TBI.

\section{PAPER 2}

\section{The Effects of Traumatic Brain Injury (TBI) in Older Adults and Cognitive Reserve}

C.E. Skilbeck, B. Coward, M. Slatyer and T. Bell

University of Tasmania, Australia

The second highest peak of TBI involves those aged 65 to 70 years, although few studies have investigated their post-TBI cognitive performance. Age-related decline in cognition is well documented in the general population, and it has been suggested that TBI may trigger accelerated ageing. Conversely, the cognitive reserve hypothesis suggests that older adults with higher IQs have a higher cognitive reserve and will show less cognitive impairment following TBI. The current study included 300+ participants, aged 16 to 75 years, assessed within 2 weeks of their TBI. Participants completed processing speed, working memory, verbal learning, and verbal fluency tasks. The NART provided an estimate of premorbid IQ. Severity of TBI was measured using PTA, and age was classified into 4 groups. The study's hypotheses were: (1) older TBI participants will show greater cognitive impairment than younger TBI participants, using ageadjusted normative scores, (2) more severe TBI will be associated with greater cognitive impairment, (3) higher IQ in older participants will be associated with less cognitive impairment. Significant effects of TBI severity were noted on many cognitive tests, and all test results showed significant premorbid IQ effects. While the cognitive reserve hypothesis received only partial support, that support was observed in relation to information processing speed (the central variable in the hypothesis). The findings demonstrate the expected effects of TBI severity, but do not generally support the hypothesis that older adults will be differentially penalised by TBI. The limited, but focussed, support for the cognitive reserve hypothesis warrants further investigation. 
CONCURRENT SESSION 6:

PSYCHOSOCIAL OUTCOMES AFTER BRAIN IMPAIRMENT IN THE ELDERLY

\title{
PAPER 1
}

\section{An Uncertain Journey}

\author{
Beryl Fabian and Jackie Summers
}

Waitemata District Health Board, Australia

\begin{abstract}
E arly recognition, diagnosis and treatment of symptoms of Alzheimer's disease (AD) affords the most promising medical, psychosocial and economic outcomes for all people affected by it. The obstacles to this ideal are not well understood. Concerns have been raised about the quality of health professionals' responses to AD. This study sought to address gaps in psychosocial understandings of $\mathrm{AD}$ by adopting case-focused, qualitative methods to research the views of 8 people diagnosed with $\mathrm{AD}$, and those of their family supporters. Specifically, attention was given to factors that mediate the subjective experience of diagnosis at the psychological level, the level of health, and within the particular social, cultural and political context of Auckland, New Zealand. An integration of within-case and across-case analyses of the interview data ensured that the unique features of each case could be honoured while a synthesis of the major themes across cases, afforded an appreciation of the common phenomenon of diagnosis for $\mathrm{AD}$. A psychosocial process was identified in participants' cognitive responses to changing ability, although they did not experience the phases of recognising, rationalising, realising and responding in the same temporal pattern. A common analytic thread was the concept of uncertainty; the data revealing that it was in the presence of greater uncertainty that participants were likely to be physically, socially and psychologically more vulnerable to the impact of AD. Recommendations for clinical practice focus on ways to ameliorate the phenomenological uncertainty inherent in the experience of discovering dementia and receiving a diagnosis of AD.
\end{abstract}

\section{PAPER 2}

\section{The Impact of Residential Respite on People With Dementia}

\author{
B. Ryburn, C. Dolye and Y. Wells \\ Lincoln Centre for Research on Ageing, Australian Institute for Primary Care, La Trobe University, \\ Australia
}

esidential respite care remains one of the most commonly available and
advocated services for family carers of people with dementia. Carers are
(e.g., an important opportunity to undertake some activities without the care-
recipient that may be important for health, wellbeing and stress relief), but they
often have ongoing concerns about its impact on the wellbeing of the care
recipient. The literature currently is not reassuring; although some studies have
been associated with a temporary diminution in the frequency of challenging
behaviours, others have been associated with an increase in challenging
behaviours and stress levels. In this paper we provide an overview and critique
of the current body of research and some suggestions for future research and
service provision. We will also present case studies to illustrate the complex-
ity of the issue, including the extent to which outcomes are likely to depend on
both individual and institution specific factors. 


\title{
PAPER 3
}

\section{A New Model for Predicting Adherence to Treatments in Poststroke Patients}

\author{
N. Coetzee ${ }^{1}$ and D.G. Andrewes ${ }^{1,2}$ \\ ${ }^{1}$ Department of Psychology, University of Melbourne, Australia \\ 2 Department of Rehabilitation, The Royal Melbourne Hospital, The Royal Park Campus, Australia
}

\begin{abstract}
A lthough the importance of adherence to treatment to prevent secondary stroke and increase recovery is well documented, the reasons for poor compliance are not understood. Past studies have also failed to measure adherence over time, even though treatments are long term. This research measured the incidence of adherence in a longitudinal study; and investigated the correlates of poor adherence according to a model. Adherence model components included age, social support, emotional and cognitive impairment, health beliefs, physical impairments, and lesion location. Adherence was measured through pill counts and rehabilitation attendance in 136 stroke patients and 119 amputee control patients aged between 23 and 93 years at 6 weeks and 9 months post discharge. Questionnaires measuring the model components were assessed using a step-down regression analysis at the 6-week assessment. Measures of the components that were able to predict adherence at 6 weeks were then assessed on the ability to predict adherence at 9 months. This approach was used to determine the model's sensitivity and specificity in predicting adherence over time. Emotional impairment was the greatest contributor to poor adherence across all ages. Stroke patients performed worse on all adherence measures when compared with amputee patients. Adherence decreased significantly over time in stroke patients. Several measures taken at 6 months were able to usefully predict adherence at 9 months, although age was not a significant contributor. The results support the implementation of the model and suggest that factors such as cognitive and emotional impairment may assist clinicians in identifying at-risk patients.
\end{abstract}

\section{PAPER 4}

\section{The Relation Between Cognitive Function and Quality of Life (QoL) in Alzheimer's Disease: Results of a Cross-Sectional Baseline Study}

\author{
Pascalle R. Bosboom and Osvaldo P. Almeida \\ University of Western Australia (UWA), School of Psychiatry and Clinical Neurosciences, Royal Perth \\ Hospital (RPH), WA Centre of Health and Ageing (WACHA), Australia
}

While it appears that quality of life (QoL) is 'measurable' in people with Alzheimer's disease (AD), the factors that mediate change in QoL remain unclear. It can be assumed that the essence of QoL, when considered as a personal evaluation of one's life, cannot be (completely) unrelated to one of the central characteristics of $\mathrm{AD}$ : cognitive deterioration. Many studies focusing on the relationship between QoL and cognition in other chronic diseases have shown significant correlations between cognitive abilities and QoL. Yet, the relationship between cognitive impairment and QoL in AD remains unclear. The aims of our two inter-related studies are: (1) to investigate the association between cognitive decline and QoL in $\mathrm{AD}$, and (2) to determine how cognitive decline contributes as predictor of changes in QoL in AD (i.e., 18-month follow-up). We will outline our first cross-sectional investigation of community dwelling people aged $65+$ 
with probable $\mathrm{AD}$ (mild to moderate) $(n=100)$. Age, gender and education matched healthy controls are recruited. Cognitive functions are being assessed with the CAMCOG-R and a comprehensive selection of neuropsychological tests. Psychological and behavioural symptoms associated with dementia are being rated according to validated, widely used, selfrated and/or proxy rated questionnaires. The QoL-AD and SEQOL (abbreviated version) are being used to measure QoL by self-report and proxy-report from two perspectives (i.e., proxy-patient and proxy-proxy). We will present the results of our cross-sectional investigation. We expect a different association between cognitive decline and QoL in AD by selfreport and by proxy-report, which will have implications for interpretation of QoL ratings in research and clinical practice.

\title{
SATURDAY MAY 3
}

CONCURRENT SESSION 7:

SCIENCE, LAW AND THE ASSESSMENT OF DECISION-MAKING CAPACITY

INVITED SPEAKER 5 (ELIZABETH MULLALY)

NO FREE PAPERS SUBSEQUENTLY

CONCURRENT SESSION 8:

THE NATURAL HISTORY OF AKINETIC RIGID SYNDROMES

INVITED SPEAKER 6 (DAVID R WILLIAMS)

NO FREE PAPERS SUBSEQUENTLY

CONCURRENT SESSION 9:

PSYCHOLOGICAL INTERVENTION AFTER ACQUIRED BRAIN INJURY

\section{PAPER 1}

\section{Acceptance and Commitment Therapy Following Acquired Brain Injury: A Useful Alternative?}

Dana Wong ${ }^{1,2}$

${ }^{1}$ Community Integration Team, Epworth Rehabilitation, Australia

2 School of Psychology, Psychiatry, and Psychological Science, Monash University, Australia

\begin{abstract}
cceptance and commitment therapy (ACT) is a mindfulness-based Acognitive-behaviour therapy, based on the goal of creating a rich and meaningful life, while learning to accept difficult internal experiences. A growing body of research has demonstrated evidence for its efficacy in a range of clinical conditions, including depression, anxiety, stress, chronic pain and illness, and substance misuse. This paper explores the application of ACT in the acquired brain injury (ABI) population, and proposes that its values-guided behavioural interventions may provide a useful and relevant therapeutic framework for the treatment of depression, anxiety, and other
\end{abstract}


psychological problems following ABI. Modifications of ACT techniques for individuals with cognitive impairments are discussed. A case example of a 34-year-old male with a traumatic brain injury sustained in a pedestrian versus train accident will be presented. The application of ACT for depression in this case resulted in an increase in subjective mood ratings, reduction in feelings of worthlessness and hopelessness, and successful achievement of a range of occupational, physical, recreational, and psychosocial goals.

\title{
PAPER 2
}

\section{Nonpharmacological Interventions for Apathy After Acquired Brain Injury}

\author{
Amanda T. Lane-Brown ${ }^{1,2}$ and Robyn L. Tate ${ }^{1,2}$ \\ ${ }^{1}$ Rehabilitation Studies Unit, University of Sydney, Australia \\ ${ }^{2}$ Royal Rehabilitation Centre, Sydney, Australia
}

\begin{abstract}
$\mathrm{A}$ pathy is a deficiency in overt behavioural, emotional and cognitive components of goal-directed behaviour. Clinically, the essential feature is decreased goal-directed activity due to a lack of motivation. It is characterised by impaired initiative, activity and lack of concern. Apathy commonly occurs after acquired brain injury (ABI). The impact is widespread, hampering physical rehabilitation, coping skills, vocational outcome, independence, and family burden. This systematic review aimed to identify and assess the efficacy of nonpharmacological treatments for apathy in adults with ABI. Relevant studies were identified by searching 6 databases. Studies were reviewed according to the following criteria: human, over 16 years, sustained an ABI (dementia, traumatic brain injury, cerebrovascular accident or encephalitis), nonpharmacological intervention for apathy, and data reported on efficacy of treatment. The methodological quality of studies was assessed. Searches yielded 1521 articles. Less than $3 \%$ of studies met criteria. Randomised controlled trials were rare. The majority of treatments were for individuals with dementia. Few were for individuals with TBI. Studies of interventions after dementia mainly dealt with increasing participation, a behavioural component of apathy. Studies of interventions after TBI mainly dealt with deficits in initiation, a cognitive component of apathy. Behavioural interventions showed some success, although overall results were varied. This review indicated there is some evidence for behavioural interventions for apathy. However, evidence is limited, particularly for individuals with TBI. This is despite the high incidence of apathy reported in the ABI population and the implications for success of rehabilitation and psychosocial outcomes, including work, interpersonal relationships and living skills.
\end{abstract}


CONCURRENT SESSION 10:

PSYCHOEMOTIONAL FUNCTIONING IN NORMAL AGEING

INVITED SPEAKER 7 (KAARIN ANSTEY)

\title{
PAPER 1
}

\section{A Meta-Analytic Review of Emotion Recognition and Ageing: Implications for Neuropsychological Models of Ageing}

\author{
Ted Ruffman, ${ }^{1}$ Julie Henry, ${ }^{2}$ Louise Phillips ${ }^{3}$ and Vicki Livingstone ${ }^{1}$ \\ ${ }^{1}$ University of Otago, New Zealand \\ 2 University of New South Wales, Australia \\ ${ }^{3}$ University of Aberdeen, New Zealand
}

\begin{abstract}
$\mathrm{W}$ e will present the results of a meta-analysis of 21 studies $(N=705$ older adults, $N=962$ younger adults) examining age differences in emotion recognition across four modalities: faces, voices, bodies/contexts, and matching of faces to voices. The results indicate that older adults have increased difficulty recognising at least some of the basic emotions (anger, sadness, fear, disgust, surprise, happiness) in each modality, with some emotions (anger and sadness) and some modalities (face-voice matching) creating particular difficulties. The predominant pattern across all emotions and modalities was of age-related decline, with the exception that there was a trend for older adults to be better than young adults at recognising disgusted facial expressions. Indeed, of the 22 comparisons, older adults were worse on 17 , there was no difference on 4 , and there was a trend for better performance on 1 . The age-related changes identified in relation to affect recognition are examined in the context of three theoretical perspectives positivity effects, general cognitive decline, and more specific neuropsychological change. We argue that the pattern of age-related change observed is most consistent with a neuropsychological model of adult ageing. In particular, there is consistent evidence for orbitofrontal involvement in the recognition of anger and sadness, and also that this is one of the first brain regions to decline in older adulthood. We speculate as to whether the brain volume changes related to emotion recognition might comprise gray or white matter changes, and also, whether reductions in neurotransmitters might account for declining emotion recognition.
\end{abstract}

\section{PAPER 2}

\section{Electromyographic Evidence for an Age-Related Delay in Facial Expression Mimicry}

Phoebe E. Bailey and Julie D. Henry

University of New South Wales, Australia

$\mathrm{R}$ elative to other facial emotions, older adults experience particular difficulty recognising anger. Since disruption of facial mimicry impairs emotion recognition, electromyography (EMG) of the corrugator supercilii (i.e., brow) muscle region was used to compare younger $(n=30)$ and older $(n=34)$ adults' reactivity to pictures of happy, neutral and angry facial expressions. The corrugator region was selected for measurement as it is more differentially responsive to affect than other regions such as the forehead and cheek, and greater corrugator activity in response to angry faces 
relative to other expressions is the most reliable means of indexing the mimicry of anger using EMG. Across both age groups angry facial expressions evoked the expected automatic mimicry response. However, despite comparable baseline levels of activity, the magnitude of older adults' corrugator response in the 200-600 ms (but not the 400-800 ms) window post stimulus-onset was reduced relative to their younger counterparts. It is of note that no previous study has used EMG to examine the automatic component of older adults' facial responding, or to examine older adults' responding to facial expression stimuli. These data, which show subtle changes in the normal automatic mimicry response in the context of late adulthood, may therefore be relevant to understanding the well-documented difficulties that older adults have decoding facial expressions of anger. However, recommendations are made for future research to directly test the extent to which age-related difficulties decoding expressions of emotion overlap with changes in the process of mimicry.

\section{CONCURRENT SESSION 11: GENETIC AND FUNCTIONAL MARKERS OF MEMORY DISORDERS}

INVITED SPEAKER 8 (CHRISTOPHER ROWE)

\section{PAPER 1}

\section{Genetic Variation: Beyond ApoE}

David Melzer

Collaborative group of MRC-CFAS, OHAP and LASA studies, Peninsula Medical School, University of Exeter, United Kingdom

Cognitive impairment in old age shows strong heritability. Recent genome wide association studies for Alzheimer's disease indicate that several variants are identifiable in addition to ApoE. In a collaborative programme, the suggested markers plus proven type 2 diabetes variants have been examined in population cohorts, namely the UK-MRC Cognitive Function and Ageing Study (including the Oxford Healthy Ageing Project) and the Longitudinal Aging Study Amsterdam (pooled $n=2768$ ). Several polymorphisms were associated with cognitive impairment in the general older population. One gene implicated (LMNA) codes for a nuclear membrane protein and is linked to apoptosis and a progeria. A second marker is in a previously unknown locus. Genetic variants are therefore generating new aetiological hypotheses. However, premature marketing of such markers as genetic tests could pose problems 


\title{
PAPER 2
}

\section{Hippocampal Brain Injury, Recall and Recognition Memory: It All Goes Together When It Goes?}

\author{
Jonathan K. Foster, ${ }^{1,2,3} 4$ Michael A. Smith, ${ }^{3}$ Johanna C. Badcock, ${ }^{5}$ Brian Levine ${ }^{6}$ \\ and Sergio Della Sala ${ }^{7}$ \\ ${ }^{1}$ School of Biomedical, Exercise and Health Sciences, Edith Cowan University, Australia \\ 2 Sir James McCusker Alzheimer's Disease Research Unit, Hollywood Private Hospital, Australia \\ ${ }^{3}$ School of Paediatrics \& Child Health, University of Western Australia, Australia \\ ${ }^{4}$ Neurosciences Unit, Health Department of Western Australia, Australia \\ ${ }^{5}$ School of Psychiatry and Clinical Neurosciences, University of Western Australia and Centre for Clinical \\ Research in Neuropsychiatry, Graylands Hospital, Australia \\ ${ }^{6}$ Rotman Research Institute, Baycrest Centre, University of Toronto, Canada \\ 7 School of Philosophy, Psychology, and Language Sciences, University of Edinburgh, Scotland
}

\begin{abstract}
$\mathrm{S}^{\mathrm{J}}$ was a high functioning individual with bilateral damage to the strucStures of the medial temporal lobe (MTL) of the brain. Structural MRI revealed severe volumetric loss to the hippocampus bilaterally. The volume of the perirhinal cortex lay within normal limits. The case of SJ is especially interesting in the light of current controversies regarding the role of the hippocampus in mediating specific elements of long-term memory (LTM). In particular, there have been recent claims that the hippocampus per se is not typically involved in the mediation of recognition memory. SJ was administered tests of recall, recognition and other neuropsychological instruments in order to evaluate his capacity across a number of neurocognitive domains. The findings obtained with SJ indicate that both recall and recognition memory were impaired in this individual. The level of impairment of SJ's recognition memory did not vary systematically according to whether the 'familiarity' or 'recollection' components of recognition memory was being differentially evaluated. These findings (1) challenge the view that the hippocampus is involved in recall memory but not typically involved in recognition memory, and (2) suggest that both 'familiarity' and 'recollection' components of recognition memory are impaired after hippocampal injury.
\end{abstract}

\section{CONCURRENT SESSION 12:}

\section{MEASUREMENT AND ISSUES OF EVIDENCE BASED PRACTICE}

\section{PAPER 1}

\section{Neuropsychological Rehabilitation: How Good is the Evidence in Dementia?}

R. Schultz, ${ }^{1}$ Michael Perdices, ${ }^{2}$ Robyn Tate, ${ }^{1}$, Skye McDonald ${ }^{3}$, Leanne Togher $^{4}$ and S. Savage ${ }^{1}$

${ }^{1}$ Rehabilitation Studies Unit, Northern Clinical School, Faculty of Medicine, University of Sydney, Australia

2 Department of Neurology, Royal North Shore Hospital, Australia

${ }^{3}$ School of Psychology, University of New South Wales, Australia

${ }^{4}$ School of Communication Sciences and Disorders, University of Sydney, Australia

$\mathrm{T}$ here is a steadily growing body of research reporting on the efficacy of nonpharmacological rehabilitation for the neuropsychological consequences of ABI. Consequently evidence based clinical practice has increasingly gained currency but it relies upon the results of well-designed and conducted research studies such as randomised controlled trials (RCTs). Evidence from other disciplines has found that methodological rigour 
among RCTs is variable (Moseley et al., 2000). The PsycBITETM database (www.psycbite.com) lists all published, empirical reports on the effectiveness of non-pharmacological interventions for the psychological consequences of ABI. The aim of this paper is to present a survey of reports indexed on PsycBITETM and to critically review their methodological quality. Whilst PsycBITETM encompasses treatments for a broad range of psychological consequences of a range of neurological conditions, the main focus of this paper will be on treatments relating to dementia and core neuropsychological domains (executive, memory, behaviour, communication and attention). In summary, when the methodological quality of the RCTs on PsycBITE ${ }^{\mathrm{TM}}$ was examined, despite there being some high quality research within the literature, the standard of research in the field of ABI needs to be significantly improved. Methods for improving methodological rigour will be discussed.

\title{
PAPER 2
}

\section{Age Differences in Neuropsycholgoical Test Performance in Chronic Psychological Disorders}

\author{
Edward Helmes \\ Department of Psychology, James Cook University, Australia
}

Tndividuals with chronic psychological disturbances may undergo psychological assessment at some point in order to document any notable cognitive deficits associated with the disorder or because of concerns related to side effects of treatments. Education level, gender, and active treatment with psychoactive medication have been shown to influence performance on tests of learning and memory. Participants are 233 people ( 70 males) with chronic psychological disorders with strict exclusion criteria to rule out those with epilepsy or reports of abnormal EEG, with evidence of cerebral infarct from brain scan, CT, or MRI imaging, and any positive signs from neurological examination of abnormal reflexes or neuromuscular or sensory defects. The mean age was 32.7 years $(S D=15.8)$, with a mean of 10.3 years of education $(S D=3.1$ years). Personality disorders and psychotic conditions were the most frequent diagnoses, with schizophrenia the single most frequently occurring condition. Several analyses are reported to explore changes in test performance with increasing age, including correlations and group comparisons of the oldest quartile (age 45 and older) against the younger group. Preliminary analyses show changes in performance on tests of motor speed and strength, complex coordinated movements (praxis), and executive functions (measures from the Wisconsin Card Sorting Test). Additional analyses report on the relative importance of age in test performance when education and medication usage are controlled. The evaluation of performance on neuropsychological tests by people with psychological disorders highlights the importance of appropriate norms and the consideration of these factors. 


\title{
PAPER 3
}

\section{Driving Awareness Questionnaire}

Lynnette Kay, Anita Bundy and Lindy Clemson

Faculty of Health Sciences, University of Sydney, Australia

\begin{abstract}
wareness of driving ability or insight is important for safe driving. A However, measuring this complex construct is difficult and previously has not been attempted. The purpose of this research was to examine the psychometric properties of the Driving Awareness Questionnaire (DrAQ). Participants $(n=91)$ referred for an occupational therapy driving assessment completed the DrAQ, which consists of 5 questions (e.g., reason for referral). Awareness of driving ability was rated as 'absent' or 'intact' based on the discrepancy (maximum of 2 points/question) between their score and the score given by the occupational therapist. This was compared with a global judgment of awareness made by the occupational therapist and driving instructor who conducted the on-road assessment. Rasch analysis was used to examine the DrAQ. There was strong evidence for construct validity indicating that DrAQ measures a single construct. Item hierarchy was logical and goodness of fit statistics revealed a good fit for 4 items. There were no differences in test results based on gender. Differential analysis yielded strong evidence for interrater reliability. However, items tended to be too easy for the most competent people, affecting precision of measurement for them. A discrepancy score of 4 on DrAQ yielded sensitivity of $84 \%$ and specificity of $94 \%$ compared with the on-road rating of awareness. The findings indicate that DrAQ has sound psychometric properties and is capable of measuring the theoretical construct of awareness of driving ability. Further items need to be included to adequately assess those with reduced awareness of their driving ability.
\end{abstract}

\section{PAPER 4}

\section{Self-Esteem After Traumatic Brain Injury: A Comparison of Two Scales}

Robyn L. Tate, Regina Schultz and Amanda Lane-Brown

Rehabilitation Studies Unit, Northern Clinical School, Faculty of Medicine, University of Sydney, Australia

\begin{abstract}
$\mathrm{A}$ lthough self-esteem (SE) is commonly acknowledged to be adversely affected after traumatic brain injury (TBI), few research efforts have been expended in this area. Two SE scales, commonly used in the general population, have been used with the TBI group: the Coopersmith Self-Esteem Inventory (CSEI) and the Rosenberg Self-Esteem Scale (RSES). The item content of the scales differs both in terms of scope and expression, raising the possibility that they do not measure exactly the same construct. The present study had two aims: (1) to compare the two SE measures with each other and (2) to explore the relationships between the SE measures and other types of measures, including mood, functional outcome and community participation. Fifty people with severe TBI who were living in the community were examined with the CSEI and RSES, along with the Depression, Anxiety and Stress Scale, Patient Competency Rating Scale, Community Integration Measure and Sydney Psychosocial Reintegration Scale. The CSEI and RSES showed moderately high intercorrelation $\left(r_{\mathrm{s}}=0.75\right)$, but differential correlation with measures of mood (RSES higher than CSEI), functional outcome (CSEI higher
\end{abstract}


than RSES), and community participation (CSEI higher than RSES). In particular, there was a high inverse correlation between the RSES and depression $\left(r_{\mathrm{s}}=0.80\right)$. Results suggested that the CSEI and RSES do not measure the same construct and are not interchangeable. The implications for clinical practice and future research directions are discussed.

\section{POSTER PRESENTATIONS}

\section{Psychiatric Screening of Patients in a Parkinson's Disease Short Stay Rehabilitation Unit}

Joel Aizenstros, Robert lansek and Margarita Makoutonina

Victorian Comprehensive Parkinson's Program (VCPP), National Parkinson Foundation Centre of Excellence, Elsternwick Private Hospital, Melbourne, Australia

Darkinson's Disease (PD) is a common progressive neurodegenerative disorder with motor and nonmotor symptoms. Neuropsychiatric disturbances contribute greatly to reduced quality of life and carer distress. Lack of identification and treatment of nonmotor domains in both general and specialist PD rehabilitation settings is often encountered in routine practice. We assessed all PD patients admitted to a short stay rehabilitation unit to determine the incidence of current neuropsychiatric symptoms and disorders. Consecutive subjects admitted to VCPP were assessed by a multidisciplinary team that included a neurologist, psychiatrist, occupational therapist, physiotherapist, speech pathologist, dietitian, social worker, and PD trained nursing staff. During a 2-week admission, motor and nonmotor domains were assessed on clinical interview, and rating scales scores that included Beck Depression Inventory, Beck Anxiety Inventory, Unified Parkinson Disease Rating Scale (UPDRS), and Folstein's Mini-Mental State Examination (MMSE) were recorded. The data was analysed for incidence of clinically significant neuropsychiatric symptoms and disorders. A total of 62 PD patients were available for analyses. Mean age was 70.5 years, and mean disease duration was 9.8 years. Incidence of neuropsychiatric disorders was anxiety $71 \%$, sleep disorders $63 \%$, depression $58 \%$, psychosis $32 \%$, cognitive impairment $26 \%$, obsessions/impulse control disorders $16 \%$, and hypomania $10 \%$. Neuropsychiatric disorders were evident in $90 \%$ of subjects, who had an average of 2.8 disorders. It was concluded that routine psychiatric assessment as part of a multidisciplinary team is required to identify and treat the very high incidence of neuropsychiatric morbidity in specialist PD rehabilitation settings.

\section{Unirhinal Olfactory Identification and Memory in Alzheimer's Disease, Mild Cognitive Impairment and Healthy Ageing}

\footnotetext{
A. Bahar-Fuchs, ${ }^{1}$ C. Rowe, ${ }^{2}$ S. Moss ${ }^{1}$ and G. Savage ${ }^{1,3}$

${ }^{1}$ School of Psychology, Psychiatry And Psychological Medicine, Monash University, Australia

2 Department of Nuclear Medicine, Austin Health, Australia

${ }^{3}$ Macquarie Centre for Cognitive Science (Maccs), Macquarie University, Australia
}

Datients with Alzheimer's disease (AD) have impaired olfactory identification as well as impaired episodic memory, and both are markers of future $\mathrm{AD}$ in preclinical cases. Interestingly, the relationship between the 
two deficits has not been examined in depth. We tested performance on olfactory identification and subsequent recognition memory for the same odours in $\mathrm{AD}$, mild cognitive impairment (MCI) and healthy ageing, in order to explore the discriminatory power of memory for smells. $14 \mathrm{AD}, 18 \mathrm{MCI}$ and 10 control participants underwent unirhinal testing of olfactory identification and memory using a modified version of the University of Pennsylvania Smell Identification Test. There were no differences in olfactory memory scores between the groups, or any interaction with nostril side. Scores of the control and MCI groups deviated significantly from chance levels, however, but the AD group's did not. Olfactory memory and identification correlated only weakly in all groups. Among the AD patients, however, a strong relationship between olfactory memory and hedonic value (odour pleasantness ratings) was observed. Contrary to expectations that $\mathrm{AD}$ patients would find the olfactory memory task particularly difficult, the task proved challenging for elderly participants regardless of diagnostic status. On our findings, prospects for olfactory memory measures as diagnostic markers in early AD or MCI appear bleak. The data do pose interesting questions about the dependence of memory on perceptual substrates, and we suggest that the impact of hedonic value of smells in $\mathrm{AD}$ only may represent degraded semantic knowledge.

\section{Memory for Music in Alzheimer's Disease: Unforgettable}

Amee Baird and Séverine Samson

Salpetriere Hospital, Paris and University of Lille 3, France

$\mathrm{O}$ ver the last few decades there have been several case reports of patients with probable Alzheimer's disease (AD) showing preserved memory for music. These studies have highlighted an intriguing phenomenon. Patients demonstrating new learning and recall of novel music are particularly noteworthy as they suggest relatively spared musical memory against a background of impaired nonmusical memory, providing support for a specialised memory system for music that appears to be distinct from verbal and visual domains. We review the current research examining musical memory in patients with $\mathrm{AD}$, including studies that have documented facilitation of memory, specifically autobiographical recall, during music listening or therapy. In keeping with models of memory described in the non-musical domain, we propose that various forms of musical memory exist, and may be differentially impaired in $\mathrm{AD}$, reflecting the pattern of neuropathological changes associated with the condition. Our synthesis of this literature reveals a dissociation between explicit and implicit musical memory functions. Explicit musical memory, or the recognition of familiar or unfamiliar melodies, is typically impaired. In contrast, implicit, specifically procedural musical memory, or the ability to play a musical instrument, can be spared in musicians with AD. Therefore, the notion that music is unforgettable in $\mathrm{AD}$ is not wholly supported. Rather, it appears that the ability to play a musical instrument may be unforgettable in some musicians with AD. The research to date has highlighted the exciting potential for music to be used in neuropsychological assessment and rehabilitation in patients with $\mathrm{AD}$ and other neurological conditions. 


\title{
Verbal Memory and Sustained Attention Deficits During Pregnancy
}

Marie-Lisa Boukarim and E. Arthur Shores

Macquarie University, Australia

Pregnant women often report memory and attention problems; however, match the severity of their cognitive complaints. There has been little research examining reasons for this discrepancy. Therefore, consistent with studies examining neurologically impaired populations, this study proposes that subtle cognitive deficits can only be detected using cognitively complex tasks. It was predicted that the decrement in verbal memory and sustained attention performance from single- to dual-task conditions would be greater for pregnant women compared to nonpregnant women. 34 pregnant women and 44 nonpregnant women participated in the study, and within each group women were randomly allocated to either the single- or dual-task conditions. Results from the study revealed a decrement in performance on measures of verbal memory from single- to dual-task conditions to be more pronounced for the pregnant women compared to the nonpregnant women. In addition, the investigation of sustained attention showed the decrement in accuracy from single- to dual-task conditions to be greater for pregnant than nonpregnant women; however, the decrement in speed from single- to dual-task conditions for the pregnant women was equal to that of the nonpregnant women. All findings were independent of age, education, IQ, depression, anxiety and stress. The findings help validate the pregnant women's perceptions of cognitive impairment. Longitudinal, within-subject studies are needed using objective tests like the dual-task paradigm that are sensitive to subtle deficits and reflect the complexity of everyday cognitive demands.

\section{Emotional Processing Abilities in Parkinson's Disease and Its Impact on Social Interactions}

\author{
Sharon Buxton and Lynette Tippett \\ University of Auckland, New Zealand
}

Dysprosody and facial mask-like appearance are clinical symptoms in Parkinson's disease (PD) that may affect social interactions. There may also be fundamental impairments in perceiving emotional processing ability in individuals with PD, which might also affect the quality of interactions. This study examined emotional processing abilities in individuals with PD and the impact of any deficits on social interactions. Previous research on how accurately emotions are perceived (from facial expressions and tone of voice) has produced conflicting results, but several studies found a deficit in some aspects of emotional processing in individuals with PD. The study explored the emotional processing ability (both perception and expression) of individuals with PD relative to age-matched controls. It then explored the impact of these difficulties with emotional processing on an individual's quality of interactions with other people. Participants were asked to recognise and produce specific emotions with facial expressions and in the tone of spoken words. A semi-structured interview assessed how satisfied individuals with PD were with their interactions with others. Findings based on the data collected so far will be presented and discussed. 


\section{Names, Faces and Places: Rehabilitation of Memory Deficits Following Herpes Simplex Encephalitis (HSE)}

Matthew Conroy, ${ }^{1}$ Julia Schmidt ${ }^{1}$ and Ashley Walker ${ }^{2}$

${ }^{1}$ Royal Rehabilitation Centre, Sydney, Australia

2 University of Western Sydney, Australia

$\mathrm{M}$ emory and language deficits are common impairments observed following an episode of HSV. Techniques such as vanishing cues, imagery and matching have been used to rehabilitate these deficits. The current study used a multiple baseline (ABACA) design and errorless learning paradigm to investigate the effect of these techniques on naming and topographical memory in a patient (LA) following an episode of HSV. Study 1: A baseline measure of LA's ability to name hospital staff was initially established. The effect of vanishing cues and matching on naming performance was then investigated. A second baseline preceded imagery training of facename associations. Study 2: A baseline measure of LA's topographical orientation was gathered prior to map training. Following a second baseline measure, the relationship between map use and imagery was assessed. To date, only the ABA component of the study has been completed. Study 1: At baseline $1 \mathrm{LA}$ was unable to name any clinician. With vanishing cues and matching training, LA was able to successfully name and match all 7 clinicians targeted. At baseline 2 she named an average of 4 clinicians. Study 2: At baseline LA was unable to locate any therapy area. During map training she was able to locate all 6 of the targeted therapy areas. At baseline 2 she located on average 4 areas. The current study will discuss the implications of the current results in regards to existing literature that imagery techniques are better than lower level strategies in compensating for memory and naming impairments following HSV.

\section{Psychoneuroimmunology: A Functional Model for Multiple Sclerosis and Other Neurological Diseases}

Gary Fulcher

MS Society of NSWIVIC, Australia

This psychoneuroimmunology (PNI) model of MS uses the coalition of genetics, immunology, endocrinology, virology, neurology, psychology and environmental factors to explain the genesis and progress of the disease. Combining the knowledge of all of these disciplines with the notions of a systemic condition called the multiple sclerosis trait (MST) and the concept that the immune system serves as a sixth sense, this model opens up possibilities of collaboration and integration as well as a wide expanse of opportunities for research, treatment and, potentially, prevention options for MS. It may also be adaptable to other neurological diseases with inflammatory components, such as stroke, Alzheimer's and Parkinson's diseases, or amyotrophic lateral sclerosis. These opportunities are outside the traditional medical and pharmaceutical approaches but inside the scientifically sound and evidence-based models of research and practice. The PNI model encourages collaborative, multidisciplinary approaches that take into account all of the abovementioned factors simultaneously in MS research and management. The paper offers an opportunity for debate and discussion on this novel approach to MS. 


\title{
Autobiographical Memory in the Elderly: Cognitive Programs to Intervene in Geriatric Depression
}

\author{
Daniela C. Gonçalves ${ }^{1,2}$ and Pedro B. Albuquerque ${ }^{2}$ \\ ${ }^{1}$ University of Queensland, Australia \\ 2 University of Minho, Portugal
}

Geriatric depression is the most common psychopathology in older

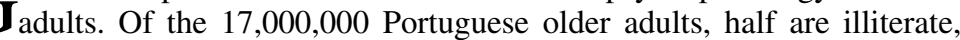
with low income and poor capacities, interfering with activities of daily living, all risk factors to develop geriatric depression (e.g., Samuelsson et al., 2005); some institutionalised and community-living Portuguese older adults already present depressive symptoms (Costa, 2005). The consequences of depression are vast and include cognitive decline and brain impairment (e.g., Katona \& Shankar, 1999, 2004). Simultaneously, the relationship between depression and autobiographical memory is well documented - while this profile is not established yet, several studies found an unequivocal relationship between depression and the inability to retrieve specific autobiographical memories (e.g., Van Vreeswijk \& Wilde, 2004; Williams \& Broadbent, 1986). Deficits in recovering specific life events are a vulnerability factor for depression (Williams, 2006), which in turn can be a risk factor for brain impairment (Gallo et al., 2003). The goal of the current study was to implement a 4-session individual program aimed at promoting specific autobiographical memories, as a way of addressing the link between autobiographical memory and depressive symptoms in an elderly population. The instruments used were Mini Mental State Examination, Geriatric Depressive Scale, Life Satisfaction Index, and Autobiographical Memory Test. The sample comprised 29 older women $(M=80.7$ years, $S D=4.5)$, with low instructional skills but no cognitive deficits (MMSE = 24). Results show an increase in both positivity and specific memories, indicating that it is possible to revert overgeneral memories. This highlights the value of autobiographical memory programs, especially for a population at an elevated risk of developing depression.

\section{Selective Preservation of the Recognition of Disgust in Early Alzheimer's Disease}

Julie D. Henry, ${ }^{1}$ Ted Ruffman, ${ }^{2}$ Skye Mcdonald, ${ }^{1}$ Marie-Andree Peek O'Leary, ${ }^{1}$

Louise H. Phillips, ${ }^{3}$ Henry Brodaty', and Peter G. Rendell ${ }^{4}$

${ }^{1}$ University of New South Wales, Australia

2 University of Otago, New Zealand

${ }^{3}$ University of Aberdeen, New Zealand

${ }^{4}$ Australian Catholic University, Australia

The neural substrates that subserve decoding of different emotional 1 expressions are subject to different rates of degeneration and atrophy in Alzheimer's disease (AD), and there is therefore reason to anticipate that a differentiated profile of affect recognition impairment may emerge. However, it remains unclear whether AD differentially affects the recognition of specific emotions. Further, there is only limited research focused on whether affect recognition deficits in $\mathrm{AD}$ generalise to ecologically valid stimuli. In the present study, relatively mild AD participants $(n=24)$, older $(n=30)$ and younger controls $(n=30)$ were administered measures of affect recognition. While significant $\mathrm{AD}$ deficits were observed relative to both the younger and older control groups on a measure that involved labeling of 
static images of facial affect, no significant $A D$ effect was observed for a more ecologically valid measure that involved dynamic displays of facial expressions, in conjunction with paralinguistic and body movement cues. These data therefore suggest that affect recognition in everyday life may be relatively preserved, at least in the earlier stages of $\mathrm{AD}$. On the static measure, significant AD deficits were observed for all emotions with the exception of disgust. The relative preservation of disgust, and the role of the basal ganglia in decoding this emotion, will be discussed.

\section{Narrative After Traumatic Brain Injury (TBI): A Comparison of Monologic and Jointly Produced Narrative}

Mikaela Jorgensen and Leanne Togher

Discipline of Speech Pathology, University of Sydney, Australia

ifficulties with narrative production have been widely noted in the literature on TBI (Coelho, 2002). While narrative does not often exist as a monologue in everyday interactions, individuals with TBI are often assessed using a monologic task or with an unfamiliar partner, usually a therapist. Socially co-constructed narratives have been targeted in children with TBI (Ylvisaker et. al., 1998) but have not yet been studied in adults with TBI. The aim of the project is to study the effects of a familiar partner on the production of narrative after TBI. 10 participants with TBI were matched with 10 control participants for sex, age, and education. Participants independently retold a story from a picture sequence and also retold a video segment with a friend. The resulting discourse was analysed for productivity, cohesion, story grammar and informational content (Cherney, 1990) as well as exchange structure in the jointly produced task (Ventola, 1987). In the jointly produced narrative, there was no significant difference in performance and participation between individuals with TBI and control participants. Participants with TBI demonstrated a significant improvement between the monologic and the jointly produced task in story grammar and informational content, but not in productivity and cohesion. Competent participation and production of narrative is possible for individuals with TBI when they engage in meaningful interactions with friends. The natural scaffolding provided by the friends of participants with TBI in the jointly produced narrative facilitated improvements in story grammar and informational content. These findings indicate an avenue for training everyday communication partners in supporting narrative skills after TBI, and for the use of jointly produced narrative as an additional assessment tool to create a holistic view of everyday skills.

\section{What Was It I Was Doing Just Now? B. Diffusion Model Parameters and Electrophysiological Correlates of Cognitive Control with Normal Ageing}

F. Karayanidis, ${ }^{1,2}$ L.R. Whitson, L.R. ${ }^{1,2}$ and P.T. Michie ${ }^{1,2}$.

${ }^{1}$ Functional Neuroimaging Laboratory, School of Psychology, University of Newcastle, Australia

${ }^{2}$ Centre for Brain and Mental Health Research, Newcastle, Australia

Tormal ageing is associated with reduced performance on executive con1 trol tasks. The excellent time resolution of event-related potentials (ERPs) was combined with diffusion model parameters of decision making in order to elucidate the cognitive processes that underlie age-related changes in executive control. In cued task-switching paradigms, increased 
cue-locked positivity emerges in preparation for an impending change in task and is associated with goal setting, whereas reduced stimulus-locked positivity is associated with task activation. Diffusion model parameters provide estimates of response conservativeness, quality of information and nondecision time. Age-related changes in ERP components and diffusion model parameters were examined in well-trained Young, Middle and Older groups. Single task and task-switching conditions were compared in a long cue-stimulus interval condition. The relationship between ERP measures and observed and latent behavioural variables was examined. In cue-locked waveforms, both Middle and Older showed less efficient differentiation between single task and repeat trials in the task-switching condition. Increased RT mixing cost in the Older group was associated with more prolonged processing of repeat trials after stimulus onset. Age-related effects were less pronounced on ERP components associated with task switching, consistent with few differences in RT switch cost. Diffusion model parameters showed that age-related increase in RT was associated with longer nondecision time and more conservative response criterion. The relationship between diffusion model parameters and ERP measures was examined to help define the processes contributing to the age-related behavioural changes. Implications for models of task-switching and cognitive ageing will be discussed.

\title{
Predictors of Subjective Cognitive Complaint After First Ever Ischaemic Stroke
}

\author{
Fiona Lamb ${ }^{1}$, Jacqueline Anderson ${ }^{1,2}$ and Michael Saling ${ }^{1,3}$ \\ ${ }^{1}$ University of Melbourne, Australia \\ 2 The Alfred Hospital, Australia \\ ${ }^{3}$ The Austin Hospital, Australia
}

$\mathrm{T}$

The subjective experience of cognitive difficulty after a first ever stroke is common, and is a significant source of distress for patients. Clinically it has been observed that even when patients are informed that their cognitive skills are intact, and that their performance on formal testing is normal, their fears are not always allayed as they continue to experience cognitive difficulties in everyday life. In order to better understand patients' experiences we need to understand the factors which underlie these difficulties. The aim of the current study therefore was to identify the factor, or factors which best predict subjective cognitive complaint after first ever stroke. It was anticipated that stroke induced fatigue, physical limitations, and affect would predict a greater proportion of the variance of subjective cognitive complaint, than objective cognitive assessment, or informant report of cognitive change. Participants aged between 50-80 years old, who had been diagnosed with their first ever ischaemic stroke were assessed between 3-6 months poststroke. Participants completed a number of measures assessing: level of fatigue, physical limitations, current affect, and subjective cognitive difficulties. All participants underwent a brief cognitive assessment and an informant report of change in cognitive functioning post stroke was collected. To our knowledge this is the first study that has investigated the important issue of complaint after first-ever stroke. The results of the study will be outlined and the relevance of these findings for the stroke clinician will discussed. 


\title{
Evidence-Based Clinical Guidelines for Mild Traumatic Brain Injury
}

Sue Lukersmith

Motor Accidents Authority NSW/Brain Injury Rehabilitation Directorate, Australia

$\mathrm{M}$ ild traumatic brain injury arising from motor vehicle accidents, falls, work/sports accidents, assaults or domestic violence is frequently undiagnosed or mismanaged - yet the majority of traumatic brain injuries are mild. There can be significant sequela which impact on the person's life and their family. In New South Wales the Brain Injury Directorate (BIRD) and the Motor Accidents Authority (MAA) commenced a joint project to develop evidence based clinical guidelines for MTBI. Over 2 years, the guidelines have been developed through the search for quality research evidence, rating the strength of the evidence to formulate recommendations. Extensive consultation occurred with representatives from key stakeholders and consensus recommendations developed where evidence was lacking. The research evidence confirms that early diagnosis and management does improve outcome. Symptoms generally resolve within 3 to 6 months. The focus of the guidelines is on recommendations and resources for clinicians in prehospital services, emergency departments and general practitioners. The guidelines emphasise the need for education and reassurance to be included in management. A patient advice sheet that incorporates key parameters was developed for use by all services. The guidelines include definitions, flow charts for the assessment and management by acute services and general practitioners. There are recommendations on screening tools for posttraumatic amnesia testing, post concussion symptoms and clinician guidance notes for the management of return to work/study, driving and sport.

\section{Cross-Linguistic Factors in Normal Age-Related Language Use: Evidence From English Versus Turkish Speakers}

\author{
Evrim March ${ }^{1,2}$ \\ ${ }^{1}$ Department of Psychology, University of Melbourne, Australia \\ 2 School of Psychology, La Trobe University, Australia
}

Deixis is a linguistic tool derived from the Greek word for 'pointing', and it handles reference in relation to the immediate communicative context. Because of its role in discourse production, deixis is a significant variable for investigating language use particularly in relation to nouns. This study compared the uses of deixis (spatial vs. person) and nouns in 26 Anglo-Australian and 26 Turkish healthy older adults over the age of 64 . Spoken language samples were obtained using the Cookie-theft picture (Goodglass \& Kaplan, 1983), and a cartoon picture (Schulz, 1976), and subjects were also administered a set of neuropsychological measures. (i.e., level of general cognitive functioning, verbal and visual working memory, language testing). Statistical analyses were undertaken to examine the group differences, the language use relationships, and the link between language use and neuropsychological measures. The findings revealed that the language-specific linguistic elements played an important role on how Turkish versus English speakers used nouns and deixis in a given communicative context. There was a general trend for higher spatial deixis in the Turkish, and higher person deixis in the English group. Furthermore, the role of cognition in language use was partially distinct across the two language groups. For example, reduced neuropsychological 
performance was linked to higher person deixis in the Turkish group, but to higher spatial deixis in the English group. The study overall demonstrates the role of the language system in the interplay between language use and cognition, and highlights the importance of cross-linguistic factors in brain-language relationships.

\title{
Psychometric Properties of the Western Neuro Sensory Stimulation Profile (WNSSP)
}

Robyn McLaclan, ${ }^{1}$ Natasha Lannin, ${ }^{2,3}$ Anne Cusick ${ }^{1}$ and Jeanine Allaous ${ }^{2}$

${ }^{1}$ University of Western Sydney, Australia

${ }^{2}$ Royal Rehabilitation Centre Sydney, Australia

${ }^{3}$ University of Sydney, Australia

There is a high rate of misdiagnosis between disorders of consciousness (DOC) with estimates of misdiagnosis of up to $45 \%$. This study examined the psychometric properties of the WNSSP, a behavioral assessment tool designed to assess individuals with DOC. The internal consistency reliability and predictive ability of the WNSSP was evaluated, and the concurrent validly of the WNSSP with the Glasgow Coma Scale (GCS) and Ranchos Los Amigos Scale of Cognitive Functioning Scale (Rancho scale) was examined. The study employed a retrospective chart audit design. Participants included 37 patients with a diagnosis of vegetative state or minimally conscious state. Main outcome measures were destination of discharge, scores obtained on the WNSSP; Rancho scale; GCS; Functional Independence Measure. Cronbach's alpha coefficients demonstrated high internal consistency of the WNSSP Total Scale (0.933). WNSSP and Rancho scale scores demonstrated a moderate correlation at admission (Spearman's rho $=0.693, p=.01$ ), and a high correlation at discharge (Spearman's rho $=0.788, p=.01$ ). No significant relationship between the WNSSP and the GCS was obtained. A weak correlation was obtained between WNSSP Total at admission and FIM Total discharge scores (Spearman's rho $=0.367, p=.01$ ). A significant, but negative correlation, between WNSSP score at admission and the participant's discharge destination was demonstrated (Spearman's rho $=-0.376, p=.01$ ). The WNSSP has high internal consistency and good concurrent validity with the Rancho scale, but limited ability to predict functional outcomes or destination of discharge of individuals with DOC.

\section{Age-Effects in Prospective Memory: Laboratory-Based Versus Naturalistic-Based Performances}

\author{
S. McLean, ${ }^{1}$ G. J. Kinsella, ${ }^{1,2}$ and B. Ong, B ${ }^{1}$ \\ ${ }^{1}$ Psychological Science, La Trobe University, Australia \\ 2 Psychology Department, Caulfield General Medical Centre, Australia
}

Drospective memory, or remembering to do things, is a potentially useful paradigm for neuropsychologists to investigate memory complaints of older adults as it is frequently identified as a common problem in the early stages of degenerative disorder. Interestingly, investigations of age-related changes in prospective memory in naturalistic settings have frequently reported that healthy older adults can outperform or at least perform as accurately as younger adults, and yet, within a laboratory setting age-related deficits are generally reported. However, prospective memory tasks in naturalistic settings have tended to be relatively simple and amenable to use of 
cues to facilitate performance. To further investigate the surprising ageadvantage in naturalistic settings, we compared the performance of an older adult group $(n=20)$ and a younger adult group $(n=20)$ on prospective memory tasks manipulated to provide two levels of complexity (simple condition, complex condition) and performed in either a laboratory or naturalistic setting. As expected, younger adults outperformed older adults in the laboratory-based tasks. However, in the naturalistic setting, in the simple condition older adults outperformed younger adults whereas in the complex condition the age advantage was no longer apparent. These prospective memory results are discussed in relation to level of motivation, strategy use and self-report of everyday memory performance. The findings support the interpretation that increases in demands on executive attention of working memory affects prospective memory performance, regardless of the experimental setting.

\title{
Perception of Communication in Ageing: Expectations and Experiences
}

Donna McNeill-Brown and Jacinta Douglas

School Of Communication Sciences, La Trobe University, Victoria, Australia

\begin{abstract}
s we grow older, physical, cognitive, and psychosocial changes affect the way we communicate. Such changes have been widely documented in the literature; however, it is not clear if community-dwelling older people perceive these changes in their communication, or younger people expect them to occur. The aim of this study was to evaluate the experiences of older people and to compare these with the expectations of younger people. Two groups of participants were recruited: 30 younger adults $(14 \mathrm{M} / 16 \mathrm{~F}$; mean age $41.24 \pm 8.55)$ and 26 older adults $(13 \mathrm{M} / 13 \mathrm{~F}$; mean age $81.34 \pm 4.37$ ). The La Trobe Communication Questionnaire was used to assess expected and experienced pragmatic difficulties as perceived by the participants. Older participants noted whether the communication problems were occurring more or less often, or at about the same rate as when they were young. Younger participants indicated whether they expected the communication difficulties to occur more or less often, or at about the same rate when they were retirement age or older. The majority of participants expected or experienced negative changes to their communication. Younger people expected more negative changes to their communication than older people reported having experienced. Participants also expected and experienced more negative changes in cognitive rather than pragmatic aspects of communication. Results are consistent with the literature, whereby linguistic (pragmatic) features are relatively preserved in ageing, whereas cognitive changes have negative impact on conversation. Results also indicated that first-hand experience rather than stereotyping, accounted for younger people's expectations in regard to their communication skills.
\end{abstract}




\title{
Is Managing Better Psychological Distress Protective of Our Cognitive Abilities?
}

Snezana Mitrovic-Tosovic, ${ }^{1}$ Pamela Melding, ${ }^{2}$ Francois Stapelberg, ${ }^{3}$ Paul Merrick, ${ }^{4}$ Richard Worrall, ${ }^{5}$ Joanna Stewart, ${ }^{6}$ Annabel Clarke, ${ }^{7}$ Leesa Rowlands, ${ }^{7}$ Jane Bryce ${ }^{7}$ and Catherine $\mathrm{Howie}^{7}$

\footnotetext{
${ }^{1}$ Waitemata District Health Board, Mental Health Services for Older People, Kingsley Mortimer Unit, New Zealand

2 University of Auckland, Department of Psychological Medicine, New Zealand

${ }^{3}$ Counties Manukau District Health Board, Department of Anaesthesia, New Zealand

${ }^{4}$ Massey University, School of Psychology, New Zealand

${ }^{5}$ Counties Manukau District Health Board, Old Age Psychiatry Service, New Zealand

${ }^{6}$ University of Auckland, School of Population Health, New Zealand

${ }^{7}$ Centre for Clinical Research and Effective Practice (CCRep), New Zealand
}

\begin{abstract}
Tn older people, higher level of chronic psychological distress is associated with increased incidence of mild cognitive impairment. Psychological distress refers to negative emotions like feeing depressed, worrying, and having negative outlook of self and future. This study examined the relationship between the level of psychological distress and cognition following orthopaedic surgery. Specifically, the study assessed whether there was postoperative cognitive decline in patients 55 and older and whether there was a difference in cognitive outcome related to the level and chronicity of psychological distress. Using an observational, community-based, longitudinal design, participants aged 55 and older who were on waiting list for elective orthopaedic knee or hip replacement surgery were assessed at 3 pre- and 3 postoperative measures over approximately 15 months. Self-report measures of depression, anxiety, perception of pain, quality of life, and cognition were administered along with cognitive measures obtained from the computerised Cognitive Drug Research Assessment System (CDR). Findings based on the data collected so far will be presented and discussed in relation to current understandings of psychological distress and cognitive status.
\end{abstract}

\section{Preferential Processing and Attentional Bias for Threat in Repetition Blindness}

Loren Mowszowski and Skye Mcdonald

School of Psychology, University Of New South Wales, Australia

$\mathrm{N}$ euroanatomical evidence suggests the human brain has dedicated neural pathways that provide rapid processing specific to threatening stimuli. Furthermore, behavioural and neuro-imaging studies have shown robust evidence for a processing bias in favour of threatening facial expressions. Such findings are, however, mainly limited to evidence from spatial tasks. The present study investigated both the processing and attentional biases for threat in a temporal task using the repetition blindness paradigm. Repetition blindness (i.e., failure to report the second instance of an identical stimulus rapidly following the first) has been reported for a wide variety of stimuli including words, objects and faces but not, to date, facial expressions. It was hypothesised that (a) repetition blindness would be found for facial expressions and (b) this effect would be diminished in the presence of threatening facial expressions. 78 participants viewed repeated and nonrepeated, threatening and nonthreatening emotional facial expressions in rapid serial visual presentation streams, verbally reporting which expressions they had seen following each trial. As expected, repeated facial expressions produced more repetition blindness than nonrepeated expressions. In support of the processing bias for threat, repetition blindness was attenuated for threatening 
expressions relative to nonthreatening expressions. These findings add a temporal perspective to the body of knowledge on threat bias, as well as enhance the repetition blindness paradigm by demonstrating the susceptibility of facial expressions to the effect. Implications for clinical populations such as TBI patients are discussed.

\title{
Positron Emission Tomography in the Differential Diagnosis of Dementia in Young Adults: A Prospective, Community-Based Study
}

Peter K Panegyres, ${ }^{1,2}$ Jeffrey M Rogers, ${ }^{1}$ Jing Shan Wu, ${ }^{3}$ Michael McCarthy, ${ }^{3,4}$ Nat Lenzo $^{3,4}$ and William Macdonald ${ }^{3,4}$

${ }^{1}$ Neurosciences Unit, Health Department of Western Australia, Perth, Australia

2 Neurodegenerative Disorders Research, Perth, Australia

${ }^{3}$ Department of Nuclear Medicine, Royal Perth Hospital, Australia

${ }^{4}$ WA PET/Cyclotron Service, Sir Charles Gairdner Hospital, Australia

\begin{abstract}
$\mathrm{T}$ The aim of this study was to evaluate the diagnostic accuracy of positron emission tomography (PET) in the differential diagnosis of young onset Alzheimer's disease (AD) and other dementias in a community-dwelling population. Patients were a prospective sample of 102 individuals presenting consecutively to a primary care centre for examination of suspected dementing diseases in younger adults. Patients were evaluated using standard clinical criteria for the diagnosis of dementia. Functional neuroimaging data was obtained and nuclear medicine physicians blind to the clinical diagnosis generated PET diagnoses. Final clinical diagnoses based on all available data were established and compared against PET diagnoses. 49 patients received a final clinical diagnosis of early-stage AD (MMSE score $20.97 \pm 5.10$ ). There were 29 non-AD-demented patients, 11 patients suffering depression and a miscellaneous group of 13 patients. The mean age of symptom onset of dementia was $60.06 \pm 4.28$ years. Among patients with $\mathrm{AD}$, the sensitivity and specificity of PET was 78\% (95\% CI: 66-90\%) and 81\% (95\% CI: $68-86 \%$ ), respectively. The specificity of PET in the differential diagnosis of other dementias, including frontotemporal dementia, was greater than $95 \%$. Recruitment methods in this study provide a sample that may be more representative of patients in the general population and indicate that PET imaging can contribute to the diagnosis of $\mathrm{AD}$ in younger adults. The high specificity of PET suggests this technique might help in the diagnosis of other forms of dementia in younger adults.
\end{abstract}

\section{Neuropsychological Outcome and Recovery from Mild TBI in Older Adults}

Bethan Potter, ${ }^{1,5}$ Glynda Kinsella, ${ }^{1,2}$ Ben Ong, ${ }^{1}$ John Olver $^{3}$ and Thomas Kossmann ${ }^{4,5}$

${ }^{1}$ Psychological Science, La Trobe University, Australia

2 Psychology, Caulfield General Medical Centre, Australia

${ }^{3}$ Bethesda Brain Injury Unit, Epworth Hospital, Australia

${ }^{4}$ Department Of Trauma Surgery, The Alfred Hospital, Australia

${ }^{5}$ National Trauma Research Institute, Australia

$\mathrm{T}$ rauma services in Australia have recorded a marked increase in the presentation of adults over the age of 65 years, a trend likely to continue given the ageing population. Recovery from trauma in older adults, including traumatic brain injury (TBI), is therefore predicted to develop as a significant public health concern. Given preexisting factors, such as lowered cognitive reserve, comorbid medical illness and neuroanatomical changes associated with the aging brain, older adults may experience a slower and 
less complete recovery from even mild TBI. This study recruited older adults admitted to The Alfred trauma service having sustained a mild TBI as a result of a traumatic accident. Participants were assessed at 2 weeks and 3 months postinjury on a range of standardised neuropsychological measures and experimental prospective memory tasks. Self-report measures of emotional distress, and everyday cognition were also administered. Findings were compared to those of age-matched trauma patients without head trauma and healthy adults living in the community. In the acute phase, both trauma groups (mild TBI, trauma control) showed significantly impaired aspects of new learning and memory and executive attention, in addition to slowed speed of information processing relative to the healthy control group. Experimental prospective memory tasks were, however, sensitive to difficulties of the mild TBI group only. These results, and those of the 3-month follow-up, are discussed in relation to trauma and ageing, and the utility of measures that require an interaction of cognitive domains rather than indexing efficiency in single domains of cognition.

\title{
Are Individuals Recovering from Mild Traumatic Brain Injury Vigilant Drivers?
}

\author{
Megan H.W. Preece, Gina M. Geffen and Mark S. Horswill \\ School Of Psychology, University of Queensland, Australia
}

\begin{abstract}
$\mathrm{M}$ ild traumatic brain injury (MTBI) is a priority for road safety research due to its high incidence, coupled with the detrimental postconcussion symptoms and the increased likelihood that individuals with MTBI will soon return to driving (as opposed to those with more severe traumatic brain injuries). Drivers' hazard perception is the ability to quickly detect potentially dangerous traffic situations or 'read the road'. It is an important component skill of driving, as several studies suggest that worse or slower hazard perception is associated with higher crash rates (e.g., Drummond, 2000; McKenna \& Crick, 1991). Hazard perception is conceptualised as being an effortful search of upcoming traffic, requiring cognitive processes such as working memory (especially the central executive), attention, and processing speed. Given that individuals with MTBI show impairment on such cognitive functions for up to 3 months postinjury, their hazard perception could also be compromised. This presentation will propose a rationale for the study of drivers' hazard perception after MTBI, and demonstrate a hazard perception test currently being used to test the effect of MTBI on this driving skill.
\end{abstract}

\section{A New Window of Opportunity: Maximising Functional Recovery 4 Years Post-CVA}

K. Pugh, R. Lobo and R. Harrington

Jacana Acquired Brain Injury Service, Northside Health Service District, Brisbane, Australia

$\mathbf{R}$ esearch findings indicating that maximal functional recovery post CVA occurs within the first 2 years are often quoted to patients and families to assist in the adjustment process. There is however, a growing body of evidence to suggest that significant functional recovery can occur a number of years postinjury. With recent increases in state and commonwealth government funding to assist young people with disabilities in aged care facilities, individuals previously denied access to rehabilitation services are now being given a second chance at rehabilitation. This single case study presentation 
will track a young woman's slow progress through acute rehabilitation, aged care and community rehabilitation services, and the significant functional recovery gained through access to the intensive slow stream rehabilitation program at Jacana ABI service 4 years postinjury. Functional assessments were completed on admission and at 3-monthly intervals by the interdisciplinary team. Throughout the admission, the subject received daily therapies with interdisciplinary team members and regular sessions with the unit social worker. On admission, the client was unable to manoeuvre an electric wheelchair due to perceptual and motor difficulties, required maximal assistance with self-care tasks, and used an alphabet board and gestures to communicate. Throughout the admission, significant improvements were achieved, with the client regaining independent wheelchair mobility, requiring only light assistance with self care tasks and able to actively participate in extensive verbal conversation. Factors facilitating improved response to rehabilitation including interdisciplinary team processes; pharmaceutical interventions; appropriate intensity, frequency and duration of individual therapy programs; environmental facilitators; and intrinsic client factors will be discussed.

\section{Emotion Regulation in Alzheimer's Disease}

Peter G. Rendell, ${ }^{1}$ Julie D. Henry, ${ }^{2}$ Amanda Sciculana ${ }^{1}$ and Michelle Jackson ${ }^{1}$

${ }^{\prime}$ School of Psychology, Australian Catholic University, Melbourne, Australia

${ }^{2}$ School of Psychology, University of New South Wales, Australia

The ability to regulate the experience and expression of emotion is an 1 important determinant of mental health, occupational effectiveness, and the formation of sustainable relationships. However, no study to date has directly assessed how this facet of socioemotional functioning is affected in relation to dementia. The present study thus set out to examine the capacity to engage in particular emotion regulatory strategies, and specifically, the ability to amplify the emotional expression of an experienced emotion ('amplification') or suppress the emotional expression of an experienced emotion ('suppression') while watching film clips selected to elicit amusement. 20 participants with Alzheimer's disease (AD) and 20 demographically matched controls were asked to watch three different amusing film clips, while engaging in different regulatory strategies. The results indicate that although no differences between the two groups were observed in self-rated amusement, individuals with AD presented with significantly reduced emotion expressive behaviour. Further, while individuals with $\mathrm{AD}$ retained the ability to use suppression to regulate emotion expressive behaviour, they were similar to controls in that they experienced difficulty amplifying emotion expressive behaviour. These data support a model of socioemotional functioning in $\mathrm{AD}$ that is characterized by both relative stability but also by losses. In particular, the finding that individuals with $\mathrm{AD}$ present with reduced emotion expressive behaviour may be relevant for caregivers who rely on such behaviours as indicators of how the person with dementia is feeling. 


\title{
A Randomised Controlled Trial of Upper Limb Motor Training After Acquired Brain Impairment
}

Leo Ross ${ }^{1}$, Lisa Harvey² and Natasha Lannin²

${ }^{1}$ Queensland Health, Australia

${ }^{2}$ Rehabilitation Studies Unit, University of Sydney, Australia

\begin{abstract}
bjective: To determine if additional daily therapy can further improve hand function in people with acquired brain impairment already receiving treatment. Design: Assessor-blinded, between groups randomised controlled trial. Setting: Rehabilitation inpatient and outpatient service. Participants: A sample of 40 people with hand impairment following stroke $(87 \%)$ or traumatic brain injury (13\%). The mean (SD) time since injury was 146 (334) days. Intervention: The experimental group received 45 minutes of intensive hand training 5 days per week, while the control group received only $10 \mathrm{~min}$ utes of the same training, 3 times per week over a 6-week period. Both groups received 20 minutes of shoulder and elbow training per day. Outcome measures: Primary outcomes were the Action Research Arm test (ARA) and summed manual muscle testing (SMMT) scores. Secondary outcomes included the Wolf Motor Function Test (WMFT), passive range of motion of wrist and finger flexors, disability of arm shoulder and hand questionnaire (DASH) and the Canadian Occupational Performance Measure (COPM). Results: The 45 minutes of additional training had a mean treatment effect on hand function and muscle strength of -6 points on the ARA (95\%CI 20-8) and $3 \%$ on the SMMT (95\% CI 10-16\%), respectively. Discussion: An extra 45 minutes of one-on-one task-specific training did not improve hand function in patients already receiving some therapy. Future research should focus on developing more effective ways of increasing patient upper limb practice intensity and duration without relying on expensive one-to-one-therapy.
\end{abstract}

\section{GPCOG: The New Best Screen for Dementia?}

Maria Scarcia ${ }^{1}$ and Amy Sellers ${ }^{2}$

${ }^{1}$ Institute of Mental Health, Townsville Health Service District, Queensland Health, Australia

${ }^{2}$ Aged Care Assessment Program, Australian Government Department of Health and Aging and James Cook University, Australia

$\mathrm{T}$ The prevalence of dementia and the importance of early diagnosis for patients and carers, stress the need for valid and reliable screening tools. The General Practitioner Assessment of Cognition (GPCOG; Brodaty et al., 2002), is administered in 5 minutes, may be used by a wide range of health professionals, and includes an informant interview. The GPCOG appears resistant to education and depression biases. This research aims to further establish the reliability and validity of the GPCOG as a superior dementia screen. 100 healthy community-dwelling elderly ( 55 years+) will be administered the GPCOG along with other tools including the MMSE, Cognistat, and CAMDEX. In addition to determining the reliability and validity of the GPCOG, demographic factors will be analysed to determine their effect on GPCOG performance. Preliminary analyses of results have supported the GPCOG as a reliable and valid instrument. Final results will include comparison with other dementia screens, and the effect of demographic variables on GPCOG scores. Valid and reliable screening tools for dementia are vital for health professionals to manage this widespread health concern. The GPCOG is destined to be one such tool, aiding in the early diagnosis and quality care of patients with dementia. 


\title{
Cognition and Performance of Everyday Tasks in Mild to Moderate Parkinson's Disease
}

Josephine L. Sinni, ${ }^{1}$ Glynda Kinsella, ${ }^{1}$ Ben Ong, ${ }^{1}$ Aileen K. Ho, ${ }^{2}$ Robert lansek ${ }^{3}$ and John Merory ${ }^{4}$

${ }^{1}$ La Trobe University, Australia

2 University of Reading, United Kingdom

${ }^{3}$ Kingston Centre, Southern Health, Melbourne, Australia

${ }^{4}$ Austin Health, Melbourne, Australia

Some research studies have reported that people with early Parkinson's disease (PD) can demonstrate executive attentional deficits and disruption of memory functions. However, the nature of the executive disturbance is far from clear, and the impact of cognitive difficulties on instrumental activities of daily living (IADLs) is largely unknown. The present investigation aimed to examine differences in executive attention and memory between medicated people with mild to moderate PD and age-matched controls, and to determine the contribution of these measures to reported performance of IADLs. Neuropsychological tests of executive attention and memory were administered to 70 participants with PD (aged 43-83 years) and 78 age-matched controls (aged 32-92 years). Caregivers evaluated IADL function. Participants with PD were more impaired than controls on the executive attentional and memory measures, however differences were nonsignificant after accounting for depression. Nevertheless, the executive attentional, memory, and affective variables significantly predicted reported IADL performance among the PD group, even after adjusting for differences in motor functioning. These findings indicate that subtle impairments exist among people with mild-moderate PD in a range of executive attentional and memory functions, although depression may at least partly contribute to these cognitive deficits. Furthermore, findings indicate that cognitive and affective variables are associated with functional impairment in PD.

\section{Reliability, Validity and Responsiveness of the Care and Needs Scale (CANS) for People With Traumatic Brain Injury}

\author{
Cheryl Soo, ${ }^{1}$ Robyn Tate, ${ }^{1,2}$ Kate Hopman, ${ }^{2}$ Marcella Forman, ${ }^{2}$ Tanya Secheny, ${ }^{2}$ \\ Vanessa Aird, ${ }^{3}$ Stuart Browne, ${ }^{3}$ Carissa Coulston, ${ }^{3}$ Louise Diffley ${ }^{4}$ and Jill Hummell ${ }^{4}$ \\ ${ }^{1}$ Rehabilitation Studies Unit, Sydney University And Royal Rehabilitation Centre Sydney, Australia \\ ${ }^{2}$ Brain Injury Rehabilitation Unit, Liverpool Hospital, Australia \\ ${ }^{3}$ Brain Injury Rehabilitation Unit, Royal Rehabilitation Centre Sydney, Australia \\ ${ }^{4}$ Brain Injury Rehabilitation Unit, Westmead Hospital, Australia
}

The Care and Needs Scale (CANS) was designed to address the lack of
suitable instruments available for measuring support needs of people
with traumatic brain injury (TBI). This research aimed to systematically
examine the reliability, validity and responsiveness of the CANS.
Participants were people with TBI aged 16 to 65 years recruited from 3
Brain Injury Rehabilitation Units in Sydney, Australia. Interrater reliability
and 1-week test-retest reliability were examined in a sample of 30 clients
from Liverpool Hospital. Reliability of proxy ratings between the client, rel-
ative and clinician was investigated in a sample of 40 clients from Royal
Rehabilitation Centre Sydney (RRCS). 70 clients from RRCS and Westmead
Hospital were recruited to examine criterion (concurrent) and construct (dis-
criminant) validity. Criterion (predictive) validity and responsiveness were
investigated in a sample of 40 inpatients from RRCS and Westmead 
Hospital. Intraclass correlation coefficients (ICC) indicated excellent interrater $(\mathrm{ICC}=.93$ to .96$)$ and test-retest $(\mathrm{ICC}=.98)$ reliability. Reliability of proxy ratings was fair to good ( $\mathrm{ICC}=.49$ to .72$)$. Evidence for concurrent validity was found with moderate correlation with other measures currently used for people with TBI ( $r_{\mathrm{s}}=-.61$ to.66). Predictive validity and responsiveness were shown with rehabilitation discharge CANS scores being able to predict functioning 6 months later. The CANS was also able to discriminate between different levels of injury severity $(p<.01)$ and functional independence $(p<.001)$. These findings add to the evidence regarding the CANS' excellent measurement properties: it is reliable, valid and responsive to changes that occur across recovery. It is concluded that the CANS is ready for routine application in clinical and research practice.

\title{
Tuberculous Meningitis: Neuropathological and Neuropsychological Aspects
}

\author{
Karen Wallace, ${ }^{1,2}$ E. Arthur Shores ${ }^{1}$ and Joseph Sandanam ${ }^{2}$ \\ ${ }^{1}$ Macquarie University, North Ryde, NSW, Australia \\ 2 St Joseph's Hospital, Auburn, NSW, Australia
}

Tuberculosis (TB) is an infectious disorder with a rapidly increasing inci1 dence, particularly in South East Asian countries. An infrequent but clinically significant consequence of this condition is Tuberculous Meningitis (TBM). The pathological mechanisms of TBM are diverse and complex. As such, the neuropsychological sequelae can vary. To date, neuropsychological descriptions of TBM have been diluted due to group analyses collapsing all types of bacterial meningitis into a single category. Bacterial meningitis is associated with deficits in short-term and working memory, psychomotor slowing, visuospatial processing deficits and symptoms of depression. Prognosis is variable but has not been described systematically in the literature. The case of a 41-year-old man with TBM will be discussed. Neuropsychologically, this man presented with an amnestic syndrome, confabulation, and source memory / time perception impairment in the postacute phase of recovery. Focused but not sustained attention was intact and fatigue was severe. Executive weaknesses were also apparent, including cognitive inflexibility, as was language disorder, including paraphasia and poor verbal (particularly category) fluency. Prognostic issues will be highlighted. The neuropathological features of this case and the mechanisms of cerebral pathology in TBM will also be discussed.

\section{Using the Large Allen's Cognitive Levels Screening Tool to Identify Functional Impairment in People With Memory Loss/ Early Dementia}

Jacqueline Wesson

Aged Care Psychiatry Department, Prince Of Wales Hospital, Randwick, Australia

Gunctional impairment is one of the criteria for the diagnosis of dementia (DSM IV). In most instances a definition of functional impairment is achieved by patient self-report of symptoms or by informant based reporting. A discrepancy exists in some patients between their subjective reports of impairments in function and the degree of impairment observed on formal neuropsychological testing. Assessment by an occupational therapist (OT) is only occasionally completed to clarify patients' abilities. Further, assessments conducted by OTs can be variable and are not usually standardised. It would be helpful to clarify the exact nature of these functional problems 
using a standardised OT assessment tool. This study aims to investigate the validity of a standardised OT assessment tool - Large Allen's Cognitive Levels Screening tool (LACL) - for accurately identifying functional impairment in older patients with cognitive impairment. Patients attending the Prince of Wales Hospital Memory Disorders Clinic had the LACL administered to them by the OT. This was the first assessment completed, prior to contact with medical staff, so as to ensure rater blindness to presenting symptoms, functional ability, and diagnosis. The LACL is a standardised leather-lacing task that examines a patient's functional cognition, specifically novel problem solving for a visuo-motor task. It is part of the Allen's Cognitive Disability Model and has a strong research background. The assessment takes between 10 and 25 minutes to administer. Preliminary data on the use of the LACL in the Memory Clinic for 13 patients over the last 12 months have indicated that there is little difference in the mean IADL score (using informant based modified Lawton \& Brody scale) and the mean LACL scores between patients who were diagnosed with mild cognitive impairment and early dementia. Use of the LACL may provide a more sensitive indication of functional impairment than informant based questionnaires, and may provide a validation of the subjective reports provided by patients.

\title{
What Was It I Was Doing Just Now? Behavioural Changes in Cognitive Control With Normal Ageing
}

\author{
L.R. Whitson, ${ }^{1,2}$ F. Karayanidis, ${ }^{1,2}$ and P.T. Michie ${ }^{1,2}$ \\ ${ }^{7}$ Functional Neuroimaging Laboratory, School Of Psychology, University Of Newcastle, Australia \\ ${ }^{2}$ Centre for Brain And Mental Health Research, Newcastle, Australia
}

We examined cognitive control processes in ageing using a cued trials switching paradigm. Young, Middle and Older participants completed 3 sessions of single task blocks and blocks of switching between letter and digit tasks. Cue-stimulus (CSI) and response-stimulus (RSI) intervals were manipulated to assess age effects on active task-set reconfiguration and passive dissipation of task-set interference components of task-switching. RT mixing cost [(repeats in switch blocks)-(single task trials)] reduced with increasing CSI and practice but remained larger in the Older group. This indicates less efficient maintenance of the representation of the previously active task-set under task-switch conditions, possibly related to a working memory deficit. Young and Middle groups showed significant decline in RT switch cost [(switch-repeat trials)] with increasing CSI in all sessions. In contrast, in the Older group, the reduction in switch cost with increasing CSI developed gradually across sessions, suggesting slower rate of development of an efficient preparation strategy for switch trials. However, in the final session, RT switch cost was smaller at both CSIs for the Older than the other groups, an effect driven largely by an age-related increase in repeat trials RT. The Older group also showed less RT switch cost than Young groups at the short RSI condition, again driven by increased RT for repeat trials. These findings suggest that slower learning of an efficient control strategy, coupled with faster degeneration of the contents of working memory, may account for cognitive control changes in normal ageing. 